\title{
A highly convergent and effective synthesis of the phytoalexin elicitor hexasaccharide
}

\author{
Wei Wang, Fanzuo Kong* \\ Research Center for Eco-Environmental Sciences, Academia Sinica, PO Box 2871, \\ Beijing 100085, People's Republic of China
}

Received 20 August 1998; revised 20 November 1998; accepted 25 November 1998

\begin{abstract}
The peracetylated hexasaccharide 1,2,4-tri- $O$-acetyl-3- $O$-(2,3,4,6-tetra- $O$-acetyl- $\beta$-D-glucopyranosyl)-6- $O$-(2,3,4-tri$O$-acetyl-6- $O$-(2,4-di- $O$-acetyl-3,6-di- $O$-(2,3,4,6-tetra- $O$-acetyl- $\beta$-D-glucopyranosyl)- $\beta$-D-glucopyranosyl)- $\beta$-D-glucopyranosyl)- $\alpha, \beta$-D-glucopyranose 21 was synthesized in a blockwise manner, employing trisaccharide trichloroacetimidate 2,4-di- $O$-acetyl-3,6-di- $O$-(2,3,4,6-tetra- $O$-acetyl- $\beta$-D-glucopyranosyl)- $\alpha$-D-glucopyranosyl trichloroacetimidate 17 as the glycosyl donor, and trisaccharide 4- $O$-acetyl-3- $O$-(2,3,4,6-tetra- $O$-acetyl- $\beta$-D-glucopyranosyl)-6- $O$-(2,3,4-tri$O$-acetyl- $\beta$-D-glucopyranosyl)-1,2- $O-(R, S)$ ethylidene- $\alpha$-D-glucopyranose 18 as the acceptor. The donor 17 and acceptor 18 were readily prepared from trisaccharides 3-O-(2,3,4,6-tetra- $O$-acetyl- $\beta$-D-glucopyranosyl)-6- $O$-(2,3,4-tri$O$-acetyl-6- $O$-chloroacetyl- $\beta$-D-glucopyranosyl)-1,2- $O$ - $(R, S)$ ethylidene- $\alpha$-D-glucopyranose 10 and 3,6-di- $O$ - $(2,3,4,6$ tetra- $O$-acetyl- $\beta$-D-glucopyranosyl)-1,2- $O-(R, S)$ ethylidene- $\alpha$-D-glucopyranose 11, respectively, which were obtained from rearrangement of orthoesters 3,4-di- $O$-acetyl-6- $O$-chloroacetyl- $\alpha$-D-glucopyranose 1,2-(3- $O$ - $(2,3,4,6$-tetra- $O$ acetyl- $\beta$-D-glucopyranosyl)-1,2- $O$ - $(R, S)$ ethylidene- $\alpha$-D-glucopyranosid-6-yl orthoacetate) 8 and 3,4,6-tri- $O$-acetyl- $\alpha$ D-glucopyranose $\quad 1,2-(3-O$-(2,3,4,6-tetra- $O$-acetyl- $\beta$-D-glucopyranosyl)-1,2- $O$ - $(R, S)$ ethylidene- $\alpha$-D-glucopyranosid6-yl orthoacetate) 9, respectively. The orthoesters were prepared from selective coupling of the disaccharide 3- $O$-(2,3,4,6-tetra- $O$-acetyl- $\beta$-D-glucopyranosyl)-1,2- $O-(R, S)$ ethylidene- $\alpha$-D-glucopyranose 4 with 'acetobromoglucose' (tetra- $O$-acetyl- $\alpha$-D-glucopyranosyl bromide) and 6- $O$-chloroacetylated 'acetobromoglucose', respectively. To confirm the selectivity of the orthoester formation and rearrangement, the disaccharide 4- $O$-acetyl-3- $O$ - $(2,3,4,6$-tetra$O$-acetyl- $\beta$-D-glucopyranosyl)-1,2- $O-(R, S)$ ethylidene- $\alpha$-D-glucopyranose 7 was prepared from 4 by selective tritylation, acetylation and detritylation. The title compound, an elicitor-active D-glucohexaose 3-O-( $\beta$-D-glucopyranosyl)6- $O$-(6- $O$-(3,6-di- $O$-( $\beta$-D-glucopyranosyl)- $\beta$-D-glucopyranosyl)- $\beta$-D-glucopyranosyl)- $\alpha, \beta-\mathrm{D}$-glucopyranose 1 , was finally obtained by Zemplén deacetylation of 21 in quantitative yield. (C) 1999 Elsevier Science Ltd. All rights reserved.
\end{abstract}

Keywords: Synthesis; Elicitor-active D-glucohexatose; Orthoester; Rearrangement

\section{Introduction}

The elicitor-active hexa- $\beta$-D-glucopyranosyl-D-glucitol, isolated from the mycelial walls of Phytophthora megasperma $f$. $s p$. glycinea, induces antibiotic phytoalexin accu-

\footnotetext{
* Corresponding author. Fax: + 86-10-62923563.
}

E-mail address: fzkong@mail.rcees.ac.cn (F. Kong) mulation in soybeans [1]. Biological assays of several oligosaccharides revealed that Dhexaglucoside is the minimum structural element required for high elicitor activity [2]. It should be noted that the $\beta$ configuration at $\mathrm{C}-1$ of the reducing end sugar is not necessary for elicitor activity [3f], and structural changes at the reducing end of the hexasaccharide only cause small effects on the activity [3k]. This 
information attracted us to synthesize hexasaccharide 1, which can be used as a valuable material for biological investigations. The syntheses of the heptasaccharide [3a-e,g,j] and the methyl and allyl glycosides of the hexasaccharide $[3 \mathrm{f}, \mathrm{h}, \mathrm{i}]$ have been reported since the discovery of the elicitor. Almost all of the syntheses involved 2- or 3-regioselectivity of the glucopyranoside with tedious separation, and the use of expensive reagents such as alkylsilyl and methoxybenzyl protective groups. In a preliminary communication we reported a new method for the highly convergent and effective synthesis of hexasaccharide 1 [4]. We present here the full account of this synthesis.

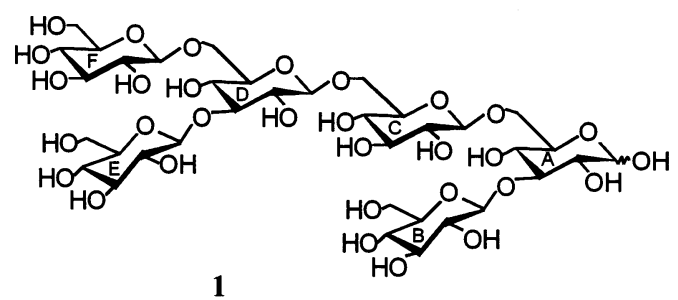

\section{Results and discussion}

Based on retrosynthetic analysis, two trisaccharide units, ABC and DEF blocks, were built as the coupling acceptor and donor. The two blocks were readily obtained from rearrangement of the corresponding orthoesters, prepared from glycosylation of disaccharide $\mathbf{4}$ with 'acetobromoglucose' (tetra- $O$-acetyl- $\alpha-\mathrm{D}-$ glucopyranosyl bromide) and 6- $O$-chloroacetylated 'acetobromoglucose' [3c], respectively. As shown in Scheme 1, 1,2-O- $(R, S)$-ethylidene4,6- $O$-benzylidene- $\alpha$-D-glucopyranoside was used as the key starting material, which was readily prepared from conventional 4,6-Obenzylidenation $[5,6]$ of $1,2-O-(R, S)$-ethylidene- $\alpha$-D-glucopyranoside [7]. Coupling [8] of 2 with acetobromoglucose in the presence of AgOTf afforded unique $\beta-(1 \rightarrow 3)$-linked disaccharide 3 as crystals in high yield (87\%). Debenzylidenation [3b] with dichloroacetic acid-water $(5: 1 \mathrm{v} / \mathrm{v})$ was carried out smoothly, furnishing crystalline diol $\mathbf{4}$ in a satisfactory yield $(90 \%)$. An attempt for selective 6-O-glycosylation of $\mathbf{4}$ with 'acetobro- moglucose' was not successful; a 4,6-di$O$-glycosylated tetrasaccharide 22 (see Scheme 2) was the major product even at low temperatures $\left(-20\right.$ to $\left.-40{ }^{\circ} \mathrm{C}\right)$ with a small quantity of the bromide donor (1 equiv). The high reactivity of the 4-OH of $\mathbf{4}$ was perhaps caused by deformation of the 1,2- $O$-ethylidene-fused pyranose ring. However, we found that the coupling of $\mathbf{4}$ with acetobromoglucose at room temperature ( $\mathrm{rt}$ ) in the presence of 2,4-lutidine (1.5 equiv), gave orthoester 9 as crystals in quantitative yield. $\mathrm{Me}_{3} \mathrm{SiOTf}$-catalyzed rearrangement [9] of 9 selectively offered the 1,6linked trisaccharide $11(76 \%)$, and acetylation gave 13. To confirm the selectivity of the rearrangement, 9 was acetylated first, then isomerized with TMSOTf to produce a compound $(74 \%)$ identical to 13 [10]. Compounds 9 and 11 were easily identified from their ${ }^{1} \mathrm{H}$ NMR spectra; the former showed seven acetyl methyl signals at $\delta 1.99-2.11$ and one upfield methyl signal of the orthoester at $\delta 1.74(\mathrm{~S})$ or $1.75(\mathrm{R})$, and the latter gave all eight methyl signals at the acetyl region $(\delta 2.00-2.17)$. Synthesis of the trisaccharide acceptor $\mathbf{1 8}$ was accomplished by the same strategy as described for the synthesis of 11 except 6-Ochloroacetylated 'acetobromoglucose' was used instead of 'acetobromoglucose'. The orthoester $\mathbf{8}$ was also crystalline, and its isomerization, acetylation, and dechloroacetylation with thiourea [11] gave the desired trisaccharide acceptor 18 as crystals. Alternative syntheses of $\mathbf{1 2}$ and $\mathbf{1 3}$ are shown in Scheme 2. Selective tritylation [12] at 6-OH of 4 (giving 5), followed by acetylation, gave $\mathbf{6}$ in high yield. Detritylation of 6 with $\mathrm{FeCl}_{3} \cdot 6 \mathrm{H}_{2} \mathrm{O}$ at $\mathrm{rt}$ furnished the disaccharide acceptor 7 with a free 6-hydroxyl without migration [13]. Coupling of 7 with 'acetobromoglucose' and 6-Ochloroacetylated 'acetobromoglucose' afforded two trisaccharides, which yielded ${ }^{1} \mathrm{H}$ NMR spectra identical to those of $\mathbf{1 3}$ and 12, respectively. This ambiguous and independent path confirmed the regioselectivity in the preparation of orthoesters 8 and 9. De-ethylidenation [3b] of 13 with $90 \% \mathrm{CF}_{3} \mathrm{COOH}$ proceeded smoothly to give crystalline 1,2-diol 14. Acetylation of 14 followed by selective deacetylation at C-1 [14], and treatment [15] with $\mathrm{CCl}_{3} \mathrm{CN} /$ DBU furnished the trisaccharide donor 17. 
Coupling [16] of $\mathbf{1 7}$ with $\mathbf{1 8}$ was promoted with $\mathrm{Me}_{3}$ SiOTf, affording the acetylated hexasaccharide 19. Zemplén deacetylation afforded the 1,2-ethylidene glucohexaose. De-ethylidenation of 19 followed by acetylation and Zemplén deacetylation [17] furnished the free hexasaccharide $\mathbf{1}$ as an amorphous solid.

In this synthesis, a new strategy of orthoester formation-rearrangement for the selective glycosylation of primary hydroxyl groups allowed the ready preparation of the related trisaccharide building blocks in large quantities. In addition, the use of acetyl, ethylidene, and benzylidene groups substantially simplified protection and deprotection steps. Furthermore, accessible materials and reagents were used and the reactions were carried out smoothly in high or good yields. Most of the intermediates involved in the synthesis were mixtures consisting of $\mathrm{R}$ and $\mathrm{S}$ isomers which were well separated and had identical reactivities. This highly convergent and effective synthesis of the title compound is suitable for large-scale preparation.

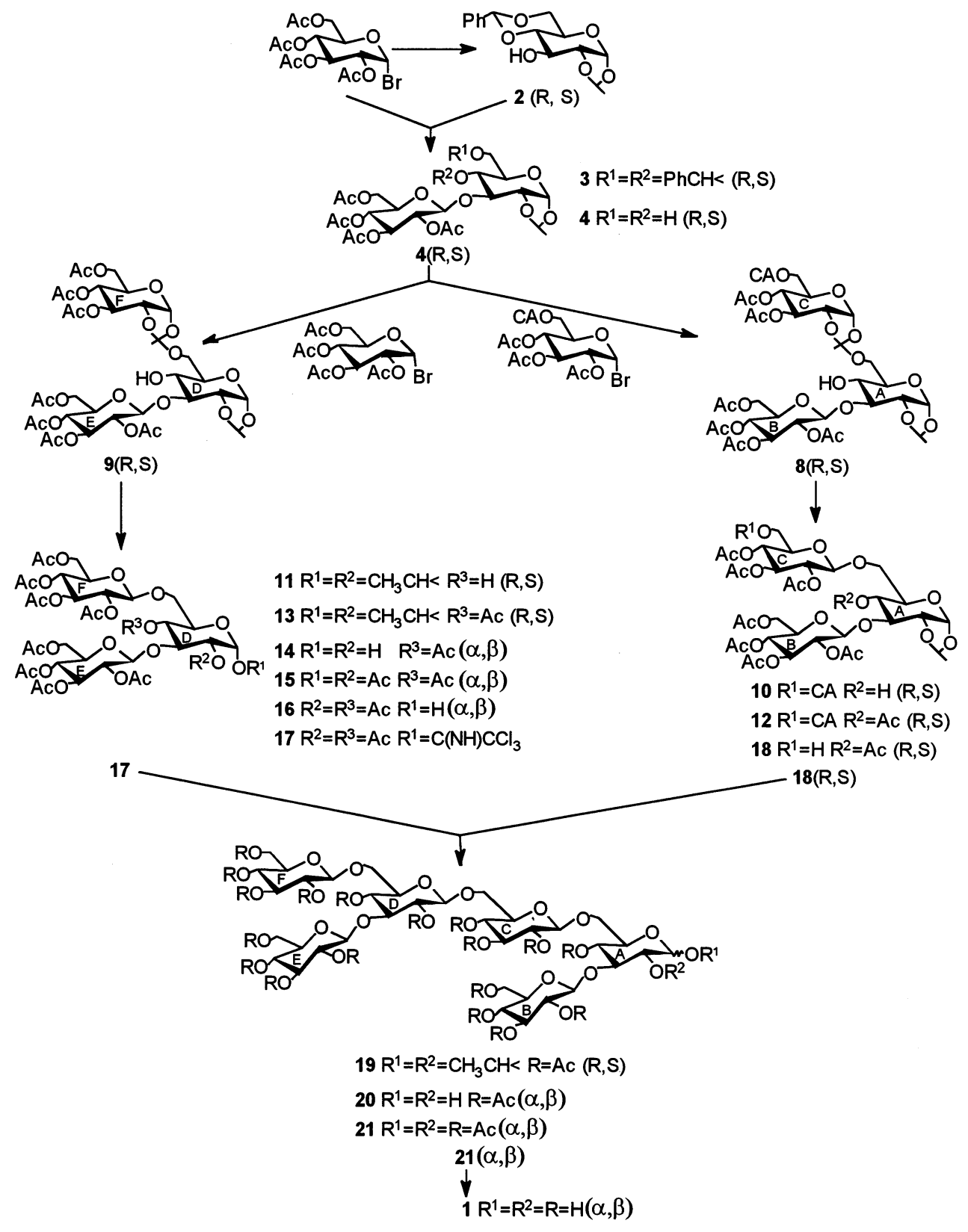

Scheme 1. 

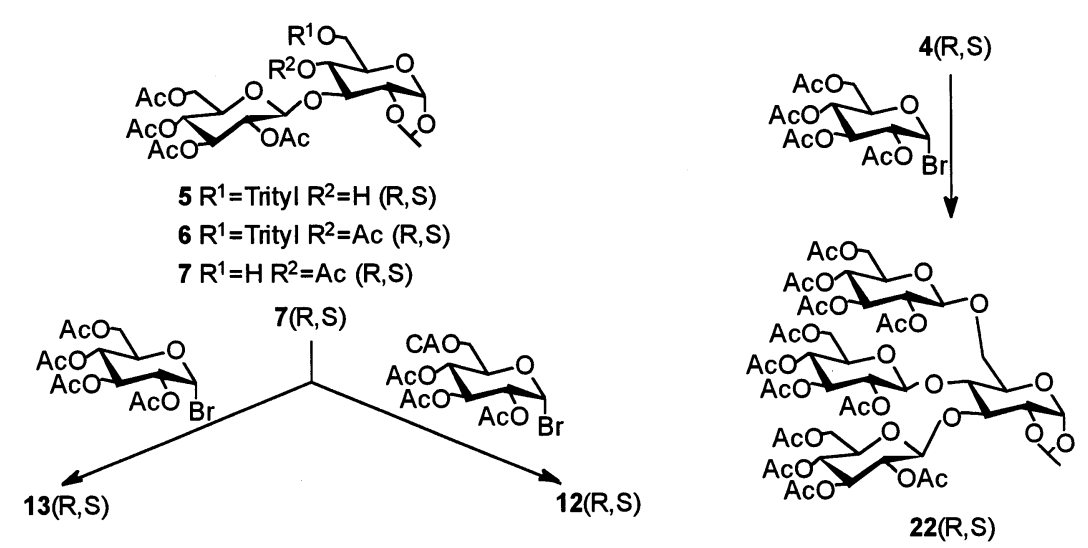

Scheme 2.

\section{Experimental}

General methods. - Melting points were determined with a 'Mel-Temp' apparatus. Optical rotations were determined with a Perkin-Elmer model 241-MC automatic polarimeter for solutions in a 1-dm, jacketed cell. ${ }^{1} \mathrm{H}$ NMR spectra were recorded with Varian XL-400 and Varian XL-200 spectrometers, for solutions in $\mathrm{CDCl}_{3}$ with $\mathrm{Me}_{4} \mathrm{Si}$ as the internal standard. Chemical shifts are expressed in ppm downfield from the internal $\mathrm{Me}_{4} \mathrm{Si} \mathrm{ab}$ sorption. $\mathrm{R}, \mathrm{S}$ isomer assignment was based on previous assignments [18] for 1,2- $O$-ethylidene sugar derivatives. Mass spectra were recorded with a VG PLATFORM mass spectrometer using the ESI technique to introduce the sample. Thin-layer chromatography (TLC) was performed on silica gel $\mathrm{HF}$, detection being affected by charring with $30 \%(\mathrm{v} / \mathrm{v})$ sulfuric acid in methanol or sometimes by UV detection. Column chromatography was conducted by elution of a column (8/100 mm, 16/240 $\mathrm{mm}, 18 / 300 \mathrm{~mm}, 35 / 400 \mathrm{~mm}$ ) of silica gel (100-200 mesh) using EtOAc/petroleum ether (bp $60-90^{\circ} \mathrm{C}$ ) as the eluent. Analytical LC was performed with a Gilson HPLC consisting of a pump (model 306), stainless steel column packed with silica gel (Spherisorb $\mathrm{SiO}_{2}, 10 \times 300 \mathrm{~mm}$ or $4.6 \times 250 \mathrm{~mm}$ ), differential refractometer (132-RI Detector), and a $\mathrm{UV}$-vis detector (model 118), and EtOAc/ petroleum ether (bp 60-90 ${ }^{\circ} \mathrm{C}$ ) was used as the eluent at a flow rate of $1-4 \mathrm{~mL} / \mathrm{min}$. Solutions were concentrated at a temperature $<60^{\circ} \mathrm{C}$ under diminished pressure.
4,6-O-Benzylidene-1,2-O-( R,S)-ethylidene- $\alpha$ D-glucopyranose (2). - According to the previously reported method [7], a solution of 'acetobromoglucose' (10 g, $24.4 \mathrm{mmol})$ in anhyd MeCN $(70 \mathrm{~mL})$ was added to tetrabutylammonium iodide (5 g, $13.6 \mathrm{mmol})$ and $\mathrm{NaBH}_{4}$ $(2.1 \mathrm{~g}, 56.8 \mathrm{mmol})$ at $0{ }^{\circ} \mathrm{C}$. After stirring for $18 \mathrm{~h}$ at $\mathrm{rt}$, TLC (2:1 petroleum ether-EtOAc) indicated the reaction was complete. Filtration and concentration of the filtrate gave a residue which was subjected to column chromatography (2:1 petroleum ether-EtOAc) to afford 2,3,4-tri- $O$-acetyl-1,2- $O$ - $(R, S)$-ethylidene- $\alpha$-Dglucopyranose $(7.3 \mathrm{~g}, 22.0 \mathrm{mmol})$. Zemplén deacetylation of the R,S mixture furnished the unprotected triol $(\mathrm{R}, \mathrm{S})$ in quantitative yield. To a solution of the triol $(\mathrm{R}, \mathrm{S})(4.5 \mathrm{~g}, 21.7$ $\mathrm{mmol})$ in $\alpha, \alpha$-diethoxytoluene $(7.6 \mathrm{~g}, 42$ $\mathrm{mmol})$, was added freshly dried $\mathrm{ZnCl}_{2}(2 \mathrm{~g})$. The mixture was stirred vigorously at $\mathrm{rt}$ for 4 h. No starting material remained by TLC $(2: 1$ petroleum ether-EtOAc) detection. $\mathrm{CH}_{2} \mathrm{Cl}_{2}$ $(30 \mathrm{~mL})$ was added to dilute the mixture. Filtration and concentration of the filtrate gave a syrup, which was purified by column chromatography $(2: 1 \quad$ petroleum etherEtOAc) to yield the title compound 2 (5.6 g, $88 \%$ ). Further purification by column chromatography (2:1 petroleum ether-EtOAc) gave the $\mathrm{R}$ and $\mathrm{S}$ isomers as crystals. For $\mathrm{R}$ isomer: $\mathrm{mp} 91-92^{\circ} \mathrm{C} ;[\alpha]_{\mathrm{D}}^{20}+70.5^{\circ}$ (c 1.1, $\left.\mathrm{CHCCl}_{3}\right)$; ${ }^{1} \mathrm{H}$ NMR: $\delta$ 7.56-7.32 (m, $5 \mathrm{H}$, $\mathrm{Ph}-\mathrm{H}), 5.53$ (s, $1 \mathrm{H}, \mathrm{PhCH}), 5.46\left(\mathrm{~d}, 1 \mathrm{H}, J_{1,2}\right.$ $4.2 \mathrm{~Hz}, \mathrm{H}-1), 5.15$ (q, $1 \mathrm{H}, J 4.4 \mathrm{~Hz}, \mathrm{CH}_{3} \mathrm{CH}$ ), $4.38\left(\mathrm{dd}, 1 \mathrm{H}, J_{1,2} 4.2 \mathrm{~Hz}, J_{2,3} 9.2 \mathrm{~Hz}, \mathrm{H}-2\right)$, 4.04-3.88 (m, 3 H, H-4, 6), 3.68 (t, 
$1 \mathrm{H}, J 9.2 \mathrm{~Hz}, \mathrm{H}-3), 3.60-3.45$ (m, $1 \mathrm{H}, \mathrm{H}-5)$, $1.48\left(\mathrm{~d}, 3 \mathrm{H}, \mathrm{J} 4.4 \mathrm{~Hz}, \mathrm{CH}_{3} \mathrm{CH}\right)$; For $\mathrm{S}$ isomer: $\operatorname{mp~} 112-114{ }^{\circ} \mathrm{C} ;[\alpha]_{\mathrm{D}}^{20}+74.5^{\circ}\left(c 1.0, \mathrm{CHCCl}_{3}\right)$; ${ }^{1} \mathrm{H}$ NMR: $\delta 5.51\left(\mathrm{~d}, 1 \mathrm{H}, J_{1.2} 4.0 \mathrm{~Hz}, \mathrm{H}-1\right)$, $5.45\left(\mathrm{q}, 1 \mathrm{H}, J 4.6 \mathrm{~Hz}, \mathrm{CH}_{3} \mathrm{CH}\right), 4.35(\mathrm{dd}, 1$ $\left.\mathrm{H}, J_{1,2} 4.0 \mathrm{~Hz}, J_{2,3} 9.8 \mathrm{~Hz}, \mathrm{H}-2\right), 4.22(\mathrm{t}, 1 \mathrm{H}$, $J 6.0 \mathrm{~Hz}, \mathrm{H}-4), 3.96\left(\mathrm{dd}, 1 \mathrm{H}, J_{2,3} 9.8 \mathrm{~Hz}, J_{3.4}\right.$ $6.0 \mathrm{~Hz}, \mathrm{H}-3), 3.50$ (t, $J 9.8 \mathrm{~Hz}, \mathrm{H}-5), 1.40$ (d, $\left.3 \mathrm{H}, J 4.6 \mathrm{~Hz}, \mathrm{CH}_{3} \mathrm{CH}\right)$. Anal. Calcd for $\mathrm{C}_{15} \mathrm{H}_{18} \mathrm{O}_{6}$ : C, 61.22; H, 6.16. Found: C, 61.37; $\mathrm{H}, 6.21$.

3-O-(2,3,4,6-Tetra-O-acetyl- $\beta$-D-glucopyranosyl) - 4,6-O - benzylidene - 1,2- O - (R,S)-ethylidene- $\alpha$-D-glucopyranose (3). - A solution of 'acetobromoglucose' (6.15 g, $15 \mathrm{mmol}), 2$ (2.95 g, $10 \mathrm{mmol})$, and $4 \AA$ activated molecular sieves $(1.5 \mathrm{~g})$ in anhyd $\mathrm{CH}_{2} \mathrm{Cl}_{2}(40 \mathrm{~mL})$ was stirred under $\mathrm{N}_{2}$ for $1 \mathrm{~h}$ at $\mathrm{rt}$ and then cooled to $-5{ }^{\circ} \mathrm{C}$. A solution of silver triflate $(3.85 \mathrm{~g}$, $15 \mathrm{mmol})$ and 2,4-lutidine (1 mL, $8.7 \mathrm{mmol})$ in 1:1 anhyd $\mathrm{CH}_{2} \mathrm{Cl}_{2}$-toluene $(15 \mathrm{~mL})$ was added dropwise with stirring for 30 min under $\mathrm{N}_{2}$ (to ensure anhydrous conditions). The mixture was stirred for $4 \mathrm{~h}$; TLC (2:1 petroleum ether-EtOAc) showed the presence of one major product. The reaction was quenched with $4 \mathrm{~mL}$ of pyridine and $20 \mathrm{~mL}$ of $10 \%$ aq $\mathrm{Na}_{2} \mathrm{~S}_{2} \mathrm{O}_{3}$, the mixture was filtered through Celite, and the solid was washed with $\mathrm{CH}_{2} \mathrm{Cl}_{2}$ $(40 \mathrm{~mL})$. The filtrate was washed sequentially with $\mathrm{N} \mathrm{HCl}(50 \mathrm{~mL})$, satd aq $\mathrm{NaHCO}_{3}(50$ $\mathrm{mL})$, and satd aq $\mathrm{NaCl}(50 \mathrm{~mL})$. The aqueous washings were re-extracted with $\mathrm{CH}_{2} \mathrm{Cl}_{2}$ (30 $\mathrm{mL}$ ), the combined organic solutions were dried and concentrated, and the residue was subjected to flash chromatography with 2:1 petroleum ether-EtOAc as the eluent to give $3(5.4 \mathrm{~g}, 87 \%)$ as a syrup. Further purification by column chromatography $(2: 1$ petroleum ether-EtOAc) gave the $\mathrm{R}$ and $\mathrm{S}$ isomers as crystals. For $\mathrm{R}$ isomer: $\mathrm{mp} 119-120^{\circ} \mathrm{C}$; $[\alpha]_{\mathrm{D}}^{20}-45.4^{\circ}\left(c 1.6, \mathrm{CHCl}_{3}\right)$; ${ }^{1} \mathrm{H}$ NMR: $\delta 7.31-$ $7.12(\mathrm{~m}, 5 \mathrm{H}, \mathrm{Ph}-\mathrm{H}), 5.58(\mathrm{~s}, 1 \mathrm{H}, \mathrm{PhCH})$, $5.48\left(\mathrm{~d}, 1 \mathrm{H}, J_{1,2} 5.1 \mathrm{~Hz}, \mathrm{H}-1\right), 5.44-4.72(\mathrm{~m}$, $\left.4 \mathrm{H}, \mathrm{H}-2^{\prime}, 3^{\prime}, 4^{\prime}, \mathrm{CH}_{3} \mathrm{CH}\right), 4.70\left(\mathrm{~d}, 1 \mathrm{H}, J_{1,2}\right.$ $\left.8.0 \mathrm{~Hz}, \mathrm{H}-1^{\prime}\right), 4.37\left(\mathrm{dd}, 1 \mathrm{H}, J_{1,2} 5.1 \mathrm{~Hz}, J_{2,3}\right.$ $10.0 \mathrm{~Hz}, \mathrm{H}-2), 4.20-3.50(\mathrm{~m}, 8 \mathrm{H}, \mathrm{H}-3,4,5$, $\left.5^{\prime}, 6,66^{\prime}\right), 2.09,2.06,2.03,2.01(4 \mathrm{~s}, 12 \mathrm{H}, 4$ $\left.\mathrm{CH}_{3} \mathrm{CO}\right), 1.50\left(\mathrm{~d}, 3 \mathrm{H}, J 5.1 \mathrm{~Hz}, \mathrm{CH}_{3} \mathrm{CH}\right)$. For S isomer: $\mathrm{mp} 128-130{ }^{\circ} \mathrm{C} ;[\alpha]_{\mathrm{D}}^{20}+66.8^{\circ}(c$ 1.7, $\left.\mathrm{CHCl}_{3}\right) ;{ }^{1} \mathrm{H}$ NMR: $\delta 5.50\left(\mathrm{~d}, 1 \mathrm{H}, J_{1,2} 4.9\right.$
$\mathrm{Hz}, \mathrm{H}-1), 5.45$ (q, $1 \mathrm{H}, J 5.3 \mathrm{~Hz}, \mathrm{CH}_{3} \mathrm{CH}$ ), $4.76\left(\mathrm{~d}, 1 \mathrm{H}, J_{1^{\prime}, 2^{\prime}} 7.8 \mathrm{~Hz}, \mathrm{H}-1^{\prime}\right), 1.41(\mathrm{~d}, 3 \mathrm{H}$, $\left.J 5.3 \mathrm{~Hz}, \mathrm{CH}_{3} \mathrm{CH}\right)$. Anal. Calcd for $\mathrm{C}_{29} \mathrm{H}_{36} \mathrm{O}_{15}$ : C, 55.77; H, 5.81. Found: C, 55.71; H, 5.90. 3-O-(2,3,4,6-Tetra-O-acetyl- $\beta$-D-glucopyranosyl)-1,2-O-(R,S)-ethylidene- $\alpha$-D-glucopyranose (4).-Compound 3 (5 g, $8 \mathrm{mmol})$ was dissolved in $\mathrm{AcOH}(30 \mathrm{~mL})$ and treated with $4: 1 \mathrm{Cl}_{2} \mathrm{CHCOOH}-\mathrm{H}_{2} \mathrm{O}(2.5 \mathrm{~mL})$ at $\mathrm{rt}$ for $6 \mathrm{~h}$, after which TLC (1:1 petroleum etherEtOAc) indicated that the reaction was complete. The reaction mixture was diluted with $\mathrm{H}_{2} \mathrm{O}$, extracted with $\mathrm{CH}_{2} \mathrm{Cl}_{2}(3 \times 50 \mathrm{~mL})$, and the organic phase was washed with satd aq $\mathrm{Na}_{2} \mathrm{CO}_{3}(3 \times 50 \mathrm{~mL})$ and $\mathrm{H}_{2} \mathrm{O}(2 \times 50 \mathrm{~mL})$, dried, and concentrated to yield crude $4(\mathrm{R}, \mathrm{S})$ $(3.9 \mathrm{~g}, 90 \%)$ as crystals. Further purification by column chromatography (1:1 petroleum ether-EtOAc) gave the $\mathrm{R}$ and $\mathrm{S}$ isomers as crystals. For $\mathrm{R}$ isomer: $\mathrm{mp} 139-140{ }^{\circ} \mathrm{C}$; $[\alpha]_{\mathrm{D}}^{20}+45.0^{\circ}\left(c 1.3, \mathrm{CHCl}_{3}\right) ;{ }^{1} \mathrm{H}$ NMR: $\delta 5.46$ $\left(\mathrm{d}, 1 \mathrm{H}, J_{1,2} 5.0 \mathrm{~Hz}, \mathrm{H}-1\right), 5.26(\mathrm{t}, 1 \mathrm{H}, J 9.5$ $\mathrm{Hz}, \mathrm{H}-3), 5.15$ (q, $1 \mathrm{H}, J 4.9 \mathrm{~Hz}, \mathrm{CH}_{3} \mathrm{CH}$ ), $5.03\left(\mathrm{t}, 2 \mathrm{H}, J 9.5 \mathrm{~Hz}, \mathrm{H}-2^{\prime}, 4^{\prime}\right), 4.65(\mathrm{~d}, 1 \mathrm{H}$, $\left.J_{1,2} 7.9 \mathrm{~Hz}, \mathrm{H}-1^{\prime}\right), 4.27(\mathrm{~d}, 1 \mathrm{H}, J 12.0 \mathrm{~Hz}$, $\left.\mathrm{H}-6_{\mathrm{a}}^{\prime}\right), 4.10\left(\mathrm{dd}, 1 \mathrm{H}, J_{1,2} 5.0 \mathrm{~Hz}, J_{2,3} 6.4 \mathrm{~Hz}\right.$, $\mathrm{H}-2), 4.10\left(\mathrm{~d}, 1 \mathrm{H}, J 12.0 \mathrm{~Hz}, \mathrm{H}-6_{\mathrm{b}}^{\prime}\right), 3.91-3.59$ (m, $\left.6 \mathrm{H}, \mathrm{H}-3,4,5,5^{\prime}, 6\right), 2.10,2.08,2.05,2.02$ $\left(4 \mathrm{~s}, 12 \mathrm{H}, 4 \mathrm{CH}_{3} \mathrm{CO}\right), 1.46(\mathrm{~d}, 3 \mathrm{H}, J 4.9 \mathrm{~Hz}$, $\mathrm{CH}_{3} \mathrm{CH}$ ); For $\mathrm{S}$ isomer: $\mathrm{mp} 170-171^{\circ} \mathrm{C}$; $[\alpha]_{\mathrm{D}}^{20}+50.3^{\circ}\left(c 1.4, \mathrm{CHCl}_{3}\right) ;{ }^{1} \mathrm{H}$ NMR: $\delta 5.50$ $\left(\mathrm{d}, 1 \mathrm{H}, J_{1,2} 4.7 \mathrm{~Hz}, \mathrm{H}-1\right), 5.42(\mathrm{q}, 1 \mathrm{H}, J 4.7$ $\left.\mathrm{Hz}, \mathrm{CH}_{3} \mathrm{CH}\right), 4.62\left(\mathrm{~d}, 1 \mathrm{H}, J_{1,2} 8.0 \mathrm{~Hz}, \mathrm{H}-1^{\prime}\right)$, $1.38\left(\mathrm{~d}, 3 \mathrm{H}, \mathrm{J} 4.7 \mathrm{~Hz}, \mathrm{CH}_{3} \mathrm{CH}\right)$. Anal. Calcd for $\mathrm{C}_{22} \mathrm{H}_{32} \mathrm{O}_{15}: \mathrm{C}, 49.25 ; \mathrm{H}, 6.01$. Found: $\mathrm{C}$, 49.11; H, 6.01.

3-O-(2,3,4,6-Tetra-O-acetyl- $\beta$-D-glucopyranosyl)-1,2-O-(R,S)-ethylidene- $6-\mathrm{O}$ - trityl- $\alpha$-Dglucopyranose (5).- According to a reported method [12], to a solution of 4 (3.2 g, 6 $\mathrm{mmol})$ in pyridine $(15 \mathrm{~mL})$ was added triphenylmethyl chloride (4.2 g, 2.5 equiv), and the mixture was stirred for $48 \mathrm{~h}$ at $\mathrm{rt}$. TLC (2:1 petroleum ether-EtOAc) showed the starting material had disappeared. The reaction mixture was poured into ice-water (50 $\mathrm{mL}$ ) and allowed to warm to rt. The aqueous layer was extracted with $\mathrm{CH}_{2} \mathrm{Cl}_{2}(3 \times 50 \mathrm{~mL})$, and the organic phase was washed sequentially with $5 \%$ aq $\mathrm{AcOH}(3 \times 30 \mathrm{~mL})$, satd aq $\mathrm{Na}_{2} \mathrm{CO}_{3} \quad(30 \mathrm{~mL})$, and $\mathrm{H}_{2} \mathrm{O} \quad(30 \mathrm{~mL})$. 
The solution was dried and concentrated to a syrup, which was subjected to column chromatography (2:1 petroleum ether-EtOAc) to yield 5 (4.4 g, 94\%) as an R,S mixture. Further purification by column chromatography (3:1 petroleum ether-EtOAc) gave the $\mathrm{R}$ and $\mathrm{S}$ isomers as syrups. For $\mathrm{R}$ isomer: $[\alpha]_{\mathrm{D}}^{20}+$ 55.4 $4^{\circ}$ c 1.7, $\mathrm{CHCl}_{3}$ ); ${ }^{1} \mathrm{H}$ NMR: $\delta$ 7.50-7.19 $(\mathrm{m}, 15 \mathrm{H}, \mathrm{Ph}-\mathrm{H}), 5.54\left(\mathrm{~d}, 1 \mathrm{H}, J_{1,2} 4.2 \mathrm{~Hz}\right.$, H-1), 5.26 (t, $\left.1 \mathrm{H}, J 9.2 \mathrm{~Hz}, \mathrm{H}-3^{\prime}\right), 5.17$ (q, 1 $\left.\mathrm{H} J 4.0 \mathrm{~Hz}, \mathrm{CH}_{3} \mathrm{CH}\right), 5.04(\mathrm{t}, 1 \mathrm{H}, J 9.2 \mathrm{~Hz}$, $\left.\mathrm{H}-2^{\prime}\right), 5.00\left(\mathrm{t}, 1 \mathrm{H}, J 9.6 \mathrm{~Hz}, \mathrm{H}-4^{\prime}\right), 4.62(\mathrm{~d}, 1$ $\left.\mathrm{H}, J_{1^{\prime}, 2^{\prime}} 8.2 \mathrm{~Hz}, \mathrm{H}-1^{\prime}\right), 4.25-4.06(\mathrm{~m}, 3 \mathrm{H}, \mathrm{H}-2$, 6'), 3.90-3.26 (m, $\left.6 \mathrm{H}, \mathrm{H}-3,4,5,5^{\prime}, 6\right), 2.08$, 2.04, 2.01, 1.92 (s, $\left.12 \mathrm{H}, 4 \mathrm{CH}_{3} \mathrm{CO}\right), 1.42$ (d, 3 $\left.\mathrm{H}, J 4.0 \mathrm{~Hz}, \mathrm{CH}_{3} \mathrm{CH}\right)$; For $\mathrm{S}$ isomer: $[\alpha]_{\mathrm{D}}^{20}+$ $\left.61.4^{\circ}(c) 1.5, \mathrm{CHCl}_{3}\right) ;{ }^{1} \mathrm{H}$ NMR: $\delta 5.66(\mathrm{~d}, 1 \mathrm{H}$, $\left.J_{1,2} 4.0 \mathrm{~Hz}, \mathrm{H}-1\right), 5.43(\mathrm{q}, 1 \mathrm{H} J 4.3 \mathrm{~Hz}$, $\left.\mathrm{CH}_{3} \mathrm{CH}\right), 4.62\left(\mathrm{~d}, 1 \mathrm{H}, J 8.0 \mathrm{~Hz}, \mathrm{H}-1^{\prime}\right), 1.37$ (d, $\left.3 \mathrm{H}, J 4.3 \mathrm{~Hz}, \mathrm{CH}_{3} \mathrm{CH}\right)$. Anal. Calcd for $\mathrm{C}_{41} \mathrm{H}_{46} \mathrm{O}_{15}: \mathrm{C}, 63.23 ; \mathrm{H}, 5.95$. Found: $\mathrm{C}, 63.51$; $\mathrm{H}, 6.02$.

4-O-Acetyl-3-O-(2,3,4,6-tetra-O-acetyl- $\beta$ D-glucopyranosyl)-1,2-O-(R,S)-ethylidene-6-Otrityl- $\alpha$-D-glucopyranose (6).-Compound 5 (4 g, $5.1 \mathrm{mmol}$ ) was dissolved in pyridine (dry, $5 \mathrm{~mL}$ ) and the solution was cooled to $0^{\circ} \mathrm{C}$. $\mathrm{Ac}_{2} \mathrm{O}(3 \mathrm{~mL})$ was added and the solution was stirred at $\mathrm{rt}$ for $4 \mathrm{~h}$. The reaction mixture was treated as described for the preparation of $\mathbf{5}$. Further purification by column chromatography ( $3: 1$ petroleum ether-EtOAc) gave the $R$ and $\mathrm{S}$ isomers as syrups. For $\mathrm{R}$ isomer: $[\alpha]_{\mathrm{D}}^{20}+58.6^{\circ}\left(c 1.1, \mathrm{CHCl}_{3}\right) ;{ }^{1} \mathrm{H}$ NMR: $\delta 7.44-$ $7.20(\mathrm{~m}, 15 \mathrm{H}, \mathrm{Ph}-\mathrm{H}), 5.64\left(\mathrm{~d}, 1 \mathrm{H}, J_{1,2} 4.5\right.$ $\mathrm{Hz}, \mathrm{H}-1), 5.29\left(\mathrm{dd}, 1 \mathrm{H}, J_{3,4} 3.0 \mathrm{~Hz}, J_{4.5} 9.7\right.$ $\mathrm{Hz}, \mathrm{H}-4), 5.24$ (t, $\left.1 \mathrm{H}, J 9.1 \mathrm{~Hz}, \mathrm{H}-3^{\prime}\right), 5.09$ (q, $\left.1 \mathrm{H}, J 4.8 \mathrm{~Hz}, \mathrm{CH}_{3} \mathrm{CH}\right), 5.05(\mathrm{t}, 1 \mathrm{H}, J 9.1 \mathrm{~Hz}$, $\left.\mathrm{H}-2^{\prime}\right), 5.00\left(\mathrm{t}, 1 \mathrm{H}, J 9.1 \mathrm{~Hz}, \mathrm{H}-4^{\prime}\right), 4.86(\mathrm{~d}, 1$ $\left.\mathrm{H}, J 7.5 \mathrm{~Hz}, \mathrm{H}-1^{\prime}\right), 4.24-3.72(\mathrm{~m}, 6 \mathrm{H}, \mathrm{H}-2,3$, $\left.5,5^{\prime}, 6^{\prime}\right), 3.30\left(\mathrm{dd}, 1 \mathrm{H}, J_{5,6 \mathrm{a}} 4.3 \mathrm{~Hz}, J_{6 \mathrm{a}, 6 \mathrm{~b}} 10.5\right.$ Hz, H-6 $), 3.10$ (dd, $J_{5,6 \mathrm{~b}} 4.3 \mathrm{~Hz}, J_{6 \mathrm{a}, 6 \mathrm{~b}} 10.5$ $\left.\mathrm{Hz}, \mathrm{H}-6_{\mathrm{b}}\right), 2.04,2.02,2.02,1.96,1.89$ (s, $15 \mathrm{H}$, $\left.5 \mathrm{CH}_{3} \mathrm{CO}\right), 1.48\left(\mathrm{~d}, \mathrm{~J} 4.8 \mathrm{~Hz}, \mathrm{CH}_{3} \mathrm{CH}\right)$; For $\mathrm{S}$ isomer: $[\alpha]_{\mathrm{D}}^{20}+71.1^{\circ}\left(c\right.$ 1.0, $\left.\mathrm{CHCl}_{3}\right) ;{ }^{1} \mathrm{H}$ NMR: $\delta 5.67\left(\mathrm{~d}, 1 \mathrm{H}, J_{1,2} 4.6 \mathrm{~Hz}, \mathrm{H}-1\right), 5.53(\mathrm{q}, 1 \mathrm{H}$, $\left.J 4.9 \mathrm{~Hz}, \mathrm{CH}_{3} \mathrm{CH}\right), 4.79(\mathrm{~d}, 1 \mathrm{H}, J 7.6 \mathrm{~Hz}$, $\left.\mathrm{H}-1^{\prime}\right), 1.39$ (d, J 4.9 Hz, $\left.\mathrm{CH}_{3} \mathrm{CH}\right)$. Anal. Calcd for $\mathrm{C}_{43} \mathrm{H}_{48} \mathrm{O}_{16}: \mathrm{C}, 62.92 ; \mathrm{H}, 5.89$. Found: $\mathrm{C}$, 63.21; H, 5.78.
4-O-Acetyl-3-O-(2,3,4,6-tetra-O-acetyl- $\beta$-Dglucopyranosyl)- $1,2-\mathrm{O}-(\mathrm{R}, \mathrm{S})$-ethylidene $-\alpha-\mathrm{D}-$ glucopyranose (7).- To a solution of 5 (4 g, $4.9 \mathrm{mmol})$ in $\mathrm{CH}_{2} \mathrm{Cl}_{2}(50 \mathrm{~mL})$ was added solid $\mathrm{FeCl}_{3} \cdot 6 \mathrm{H}_{2} \mathrm{O}(2.7 \mathrm{~g}, 2$ equiv). The mixture was stirred at $\mathrm{rt}$ for $5 \mathrm{~h}$, at the end of which time the reaction was complete as indicated by TLC (2:1 petroleum ether-EtOAc). Water was added, and the mixture was diluted with $\mathrm{CH}_{2} \mathrm{Cl}_{2}$. The organic layers were combined, dried, and concentrated to give $7(2.4 \mathrm{~g}, 83 \%)$ as a syrup. Further purification by column chromatography $(2: 1$ petroleum etherEtOAc) gave the $\mathrm{R}$ and $\mathrm{S}$ isomers as crystals. For $\mathrm{R}$ isomer: $\mathrm{mp} 96-98^{\circ} \mathrm{C} ;[\alpha]_{\mathrm{D}}^{20}+40.1^{\circ}(c$ 2.0, $\left.\mathrm{CHCl}_{3}\right) ;{ }^{1} \mathrm{H}$ NMR: $\delta 5.57\left(\mathrm{~d}, 1 \mathrm{H}, J_{1,2} 5.1\right.$ $\mathrm{Hz}, \mathrm{H}-1), 5.22\left(\mathrm{dd}, 1 \mathrm{H}, J_{3,4} 2.3 \mathrm{~Hz}, J_{4,5} 9.1\right.$ $\mathrm{Hz}, \mathrm{H}-4), 5.21\left(\mathrm{t}, 1 \mathrm{H}, J 9.2 \mathrm{~Hz}, \mathrm{H}-3^{\prime}\right), 5.11(\mathrm{q}$, $\left.1 \mathrm{H}, J 5.4 \mathrm{~Hz}, \mathrm{CH}_{3} \mathrm{CH}\right), 5.08(\mathrm{t}, 1 \mathrm{H}, J 9.2 \mathrm{~Hz}$, H-2'), 4.98 (t, $\left.1 \mathrm{H}, J 9.2 \mathrm{~Hz}, \mathrm{H}-4^{\prime}\right), 4.83$ (d, 1 $\left.\mathrm{H}, J_{1^{\prime}, 2^{\prime}}, 7.8 \mathrm{~Hz}, \mathrm{H}-1^{\prime}\right), 4.24-3.80(\mathrm{~m}, 6 \mathrm{H}, \mathrm{H}-2$, $\left.3,5,5^{\prime}, 6^{\prime}\right), 3.75\left(\mathrm{dd}, 1 \mathrm{H}, J_{5,6 \mathrm{a}} 5.1 \mathrm{~Hz}, J_{6 \mathrm{a}, 6 \mathrm{~b}}\right.$ $\left.12.4 \mathrm{~Hz}, \mathrm{H}-6_{\mathrm{a}}\right), 3.62\left(\mathrm{dd}, 1 \mathrm{H}, J_{5,6 \mathrm{~b}} 5.1 \mathrm{~Hz}\right.$, $\left.J_{6 \mathrm{a}, 6 \mathrm{~b}} 12.4 \mathrm{~Hz}, \mathrm{H}-6_{\mathrm{b}}\right), 2.13,2.10,2.07,2.05$, 2.03 (s, $\left.15 \mathrm{H}, 5 \mathrm{CH}_{3} \mathrm{CO}\right), 1.49$ (d, $3 \mathrm{H}, J 5.4$ $\mathrm{Hz}, \mathrm{CH}_{3} \mathrm{CH}$ ); For $\mathrm{S}$ isomer: $\mathrm{mp} 110-112^{\circ} \mathrm{C}$; $[\alpha]_{\mathrm{D}}^{20}+47.4^{\circ}\left(c\right.$ 2.1, $\left.\mathrm{CHCl}_{3}\right) ;{ }^{1} \mathrm{H}$ NMR: $\delta 5.59$ $\left(\mathrm{q}, 1 \mathrm{H}, J 5.2 \mathrm{~Hz}, \mathrm{CH}_{3} \mathrm{CH}\right), 5.58\left(\mathrm{~d}, 1 \mathrm{H}, J_{1,2}\right.$ $5.1 \mathrm{~Hz}, \mathrm{H}-1), 4.84\left(\mathrm{~d}, 1 \mathrm{H}, J_{1^{\prime}, 2^{\prime}} 7.8 \mathrm{~Hz}, \mathrm{H}-1^{\prime}\right)$, $1.37\left(\mathrm{~d}, 3 \mathrm{H}, J 5.2 \mathrm{~Hz}, \mathrm{CH}_{3} \mathrm{CH}\right)$. Anal. Calcd for $\mathrm{C}_{24} \mathrm{H}_{34} \mathrm{O}_{16}: \mathrm{C}, 49.83 ; \mathrm{H}, 5.92$. Found: $\mathrm{C}$, 49.71; H, 5.98.

3,4-Di-O-acetyl-6-O-chloroacetyl- $\alpha$-D-glucopyranose 1,2-(3-O-(2,3,4,6-tetra-O-acetyl- $\beta$ $\mathrm{D}$-glucopyranosyl)-1,2-O-(R,S)-ethylidene- $\alpha-\mathrm{D}-$ glucopyranosid-6-yl orthoacetate) (8).-To a stirred mixture of 4 (1.07 g, $2.0 \mathrm{mmol}), 6-O$ chloroacetylated 'acetobromoglucose' $(1.07 \mathrm{~g}$, $2.4 \mathrm{mmol}), 2,4$-lutidine $(270 \mu \mathrm{L}, 2.4 \mathrm{mmol})$, and $4 \AA$ molecular sieves $(2 \mathrm{~g})$ in $\mathrm{CH}_{2} \mathrm{Cl}_{2}$ (dry, $30 \mathrm{~mL})$ was added silver triflate $(0.617 \mathrm{~g}, 2.4$ $\mathrm{mmol}$ ) under a nitrogen atmosphere in a dark room. The reaction was carried out at $\mathrm{rt}$ and monitored by TLC (1:1 petroleum etherEtOAc). After completion of the reaction, the mixture was partitioned between $\mathrm{CH}_{2} \mathrm{Cl}_{2}$ (40 $\mathrm{mL})$ and $\mathrm{H}_{2} \mathrm{O}(40 \mathrm{~mL})$ and the organic phase was washed with $10 \%$ aq $\mathrm{Na}_{2} \mathrm{~S}_{2} \mathrm{O}_{3}(20 \mathrm{~mL})$ and aq $\mathrm{NaCl}(30 \mathrm{~mL})$ and concentrated under reduced pressure. The residual oil was purified by column chromatography with $1: 1$ petro- 
leum ether-EtOAc as the eluent giving the product $8(\mathrm{R}, \mathrm{S})$ as an amorphous solid in $94 \%$ yield $(1.7 \mathrm{~g})$; this crude product could be used directly for further rearrangement. Further purification of $\mathbf{8}$ by HPLC (1:1 petroleum ether-EtOAc) gave the $\mathrm{R}$ and $\mathrm{S}$ isomers as crystals. For $\mathrm{R}$ isomer: $\mathrm{mp} 74-75^{\circ} \mathrm{C} ;[\alpha]_{\mathrm{D}}^{20}-$ $3.5^{\circ}\left(c 1.1, \mathrm{CHCl}_{3}\right) ;{ }^{1} \mathrm{H}$ NMR: $\delta 5.72(\mathrm{~d}, 1 \mathrm{H}$, $\left.J_{1,2} 5.8 \mathrm{~Hz}, \mathrm{H}-1_{\mathrm{C}}\right), 5.57(\mathrm{q}, 1 \mathrm{H}, J 5.2 \mathrm{~Hz}$, $\left.\mathrm{CH}_{3} \mathrm{CH}\right), 5.53\left(\mathrm{~d}, 1 \mathrm{H}, J_{1,2} 5.2 \mathrm{~Hz}, \mathrm{H}-1_{\mathrm{A}}\right), 4.68$ $\left(\mathrm{d}, 1 \mathrm{H}, J_{1,2} 7.9 \mathrm{~Hz}, \mathrm{H}-1_{\mathrm{B}}\right), 4.13(\mathrm{~s}, 2 \mathrm{H}$, $\left.\mathrm{CH}_{2} \mathrm{ClCO}\right), 2.13-2.04\left(6 \mathrm{~s}, 18 \mathrm{H}, 6 \mathrm{CH}_{3} \mathrm{CO}\right)$, $1.75\left(\mathrm{~s}, 3 \mathrm{H}, \mathrm{CH}_{3} \mathrm{CO}_{3}\right), 1.48(\mathrm{~d}, 3 \mathrm{H}, J 5.2 \mathrm{~Hz}$, $\mathrm{CH}_{3} \mathrm{CH}$ ); For $\mathrm{S}$ isomer: $\mathrm{mp} 70-72{ }^{\circ} \mathrm{C}$; $[\alpha]_{\mathrm{D}}^{20}+$ $\left.4.5^{\circ}(c) 1.5, \mathrm{CHCl}_{3}\right) ;{ }^{1} \mathrm{H}$ NMR: $\delta 5.73(\mathrm{~d}, 1 \mathrm{H}$, $\left.J_{1,2} 5.1 \mathrm{~Hz}, \mathrm{H}-1_{\mathrm{C}}\right), 5.51\left(\mathrm{~d}, 1 \mathrm{H}, J_{1,2} 4.6 \mathrm{~Hz}\right.$, $\left.\mathrm{H}-1_{\mathrm{A}}\right), 5.42\left(\mathrm{q}, 1 \mathrm{H}, J 4.6 \mathrm{~Hz}, \mathrm{CH}_{3} \mathrm{CH}\right), 4.66$ $\left(\mathrm{d}, 1 \mathrm{H}, J_{1,2} 8.0 \mathrm{~Hz}, \mathrm{H}-1_{\mathrm{B}}\right), 4.10(\mathrm{~s}, 2 \mathrm{H}$, $\left.\mathrm{CH}_{2} \mathrm{ClCO}\right), 1.39\left(\mathrm{~d}, 3 \mathrm{H}, J 4.6 \mathrm{~Hz}, \mathrm{CH}_{3} \mathrm{CH}\right)$. Anal. Calcd for $\mathrm{C}_{36} \mathrm{H}_{49} \mathrm{O}_{24} \mathrm{Cl}$ : C, 47.98; H, 5.48. Found: $\mathrm{C}, 47.82 ; \mathrm{H}, 5.44$.

3,4,6-Tri-O-acetyl- $\alpha$-D-glucopyranose 1,2-(3$\mathrm{O}-(2,3,4,6$-tetra-O-acetyl- $\beta$-D-glucopyranosyl)1,2-O-(R,S)-ethylidene- $\alpha$-D-glucopyranosid- 6 $y l$ orthoacetate) (9).- As described in the preparation of 8 , the coupling of compound $\mathbf{4}$ $(1.0 \mathrm{~g}, 1.9 \mathrm{mmol})$ with 'acetobromoglucose' (0.94 g, $2.3 \mathrm{mmol})$ was carried out in the presence of silver triflate $(590 \mathrm{mg}, 2.3 \mathrm{mmol})$ and lutidine $(265 \mu \mathrm{L}, 2.3 \mathrm{mmol})$ to furnish 9 $(\mathrm{R}, \mathrm{S})(1.58 \mathrm{~g}, 96 \%)$ as an amorphous solid. Further purification by HPLC (1:1 petroleum ether-EtOAc) gave the $\mathrm{R}$ and $\mathrm{S}$ isomers as crystals. For R isomer: $\mathrm{mp} 67-68^{\circ} \mathrm{C} ;[\alpha]_{\mathrm{D}}^{20}+$ $\left.43.7^{\circ}(c) 1.3, \mathrm{CHCl}_{3}\right) ;{ }^{1} \mathrm{H}$ NMR: $\delta 5.73(\mathrm{~d}, 1 \mathrm{H}$, $\left.J_{1,2} 5.1 \mathrm{~Hz}, \mathrm{H}-1_{\mathrm{F}}\right), 5.49\left(\mathrm{~d}, 1 \mathrm{H}, J_{1,2} 4.9 \mathrm{~Hz}\right.$, $\left.\mathrm{H}-1_{\mathrm{D}}\right), 5.40\left(\mathrm{q}, 1 \mathrm{H}, J 4.9 \mathrm{~Hz}, \mathrm{CH}_{3} \mathrm{C} H\right), 4.64$ $\left(\mathrm{d}, 1 \mathrm{H}, J_{1,2} 7.8 \mathrm{~Hz}, \mathrm{H}-1_{\mathrm{E}}\right), 2.11-2.03(7 \mathrm{~s}, 21$ $\left.\mathrm{H}, 7 \mathrm{CH}_{3} \mathrm{CO}\right), 1.74\left(\mathrm{~s}, 3 \mathrm{H}, \mathrm{CH}_{3} \mathrm{CO}_{3}\right), 1.36(\mathrm{~d}$, $3 \mathrm{H}, J 4.9 \mathrm{~Hz}, \mathrm{CH}_{3} \mathrm{CH}$ ); For $\mathrm{S}$ isomer: $\mathrm{mp}$ $78-80{ }^{\circ} \mathrm{C} ;[\alpha]_{\mathrm{D}}^{20}+49.3^{\circ}\left(c \quad 0.8, \mathrm{CHCl}_{3}\right) ;{ }^{1} \mathrm{H}$ NMR $\delta 5.74\left(\mathrm{~d}, 1 \mathrm{H}, J_{1,2} 5.1 \mathrm{~Hz}, \mathrm{H}-1_{\mathrm{F}}\right), 5.50$ $\left(\mathrm{d}, 1 \mathrm{H}, J_{1,2} 4.7 \mathrm{~Hz}, \mathrm{H}-1_{\mathrm{D}}\right), 5.41(\mathrm{q}, 1 \mathrm{H}, J 4.9$ $\left.\mathrm{Hz}, \mathrm{CH}_{3} \mathrm{CH}\right), 4.63\left(\mathrm{~d}, 1 \mathrm{H}, J_{1,2} 7.8 \mathrm{~Hz}, \mathrm{H}-1_{\mathrm{E}}\right)$, $1.35\left(\mathrm{~d}, 3 \mathrm{H}, \mathrm{J} 4.9 \mathrm{~Hz}, \mathrm{CH}_{3} \mathrm{CH}\right)$. Anal. Calcd for $\mathrm{C}_{36} \mathrm{H}_{50} \mathrm{O}_{24}: \mathrm{C}, 49.89 ; \mathrm{H}, 5.81$. Found: $\mathrm{C}$, $50.08 ; \mathrm{H}, 5.70$.

3-O-(2,3,4,6-Tetra-O-acetyl- $\beta$-D-glucopyranosyl) - 6-O - (2,3,4- tri- O - acetyl - 6-O - chloro acetyl- $\beta$-D-glucopyranosyl)-1,2-O-(R,S)-ethylidene- $\alpha$-D-glucopyranose (10). - To a stirred mixture of $8(720 \mathrm{mg}, 0.80 \mathrm{mmol})$ and $4 \AA$ molecular sieves $(0.5 \mathrm{~g})$ in $\mathrm{CH}_{2} \mathrm{Cl}_{2}$ (dry, 20 $\mathrm{mL}$ ) was added TMSOTf ( $14 \mu \mathrm{L}, 0.1$ equiv) at $0{ }^{\circ} \mathrm{C}$ under a nitrogen atmosphere. The reaction was monitored by TLC (1:1 petroleum ether-EtOAc). After completion of the reaction, triethylamine $(15 \mu \mathrm{L})$ was added to the mixture and the reaction was allowed to warm to rt. The mixture was filtered and the filtrate was treated with $\mathrm{N} \mathrm{HCl}(20 \mathrm{~mL})$, satd aq $\mathrm{Na}_{2} \mathrm{CO}_{3}(20 \mathrm{~mL})$, and $\mathrm{H}_{2} \mathrm{O}(20 \mathrm{~mL})$, dried, and concentrated. The residue was subjected to column chromatography with 1:1 petroleum ether-EtOAc as the eluent, giving product $10(\mathrm{R}, \mathrm{S})$ as a syrup in $72 \%$ yield $(518$ mg). Further purification by HPLC (1:1 petroleum ether-EtOAc) gave the $\mathrm{R}$ and $\mathrm{S}$ isomers as syrups. For $\mathrm{R}$ isomer: $[\alpha]_{\mathrm{D}}^{20}-8.3^{\circ}$ (c $\left.0.8, \mathrm{CHCl}_{3}\right) ;{ }^{1} \mathrm{H}$ NMR $\delta 5.50\left(\mathrm{~d}, 1 \mathrm{H}, J_{1,2}\right.$ $\left.4.7 \mathrm{~Hz}, \mathrm{H}-1_{\mathrm{A}}\right), 5.20(\mathrm{q}, 1 \mathrm{H}, J 4.7 \mathrm{~Hz}$, $\left.\mathrm{CH}_{3} \mathrm{CH}\right), 4.80\left(\mathrm{~d}, 1 \mathrm{H}, J_{1,2} 8.0 \mathrm{~Hz}, \mathrm{H}-1_{\mathrm{C}}\right), 4.64$ $\left(\mathrm{d}, 1 \mathrm{H}, J_{1,2} 8.0 \mathrm{~Hz}, \mathrm{H}-1_{\mathrm{B}}\right), 4.11(\mathrm{~s}, 2 \mathrm{H}$, $\left.\mathrm{CH}_{2} \mathrm{ClCO}\right), 2.07-1.99\left(7 \mathrm{~s}, 21 \mathrm{H}, 7 \mathrm{CH}_{3} \mathrm{CO}\right)$, $1.47\left(\mathrm{~d}, 3 \mathrm{H}, J 4.7 \mathrm{~Hz}, \mathrm{CH}_{3} \mathrm{CH}\right)$; For $\mathrm{S}$ isomer: $[\alpha]_{\mathrm{D}}^{20}+3.8^{\circ}\left(c 0.8, \mathrm{CHCl}_{3}\right) ;{ }^{1} \mathrm{H}$ NMR: $\delta 5.62$ $\left(\mathrm{d}, 1 \mathrm{H}, J_{1,2} 4.4 \mathrm{~Hz}, \mathrm{H}-1_{\mathrm{A}}\right), 5.50(\mathrm{q}, 1 \mathrm{H}, J 4.4$ $\left.\mathrm{Hz}, \mathrm{CH}_{3} \mathrm{CH}\right), 4.79\left(\mathrm{~d}, 1 \mathrm{H}, J_{1,2} 7.8 \mathrm{~Hz}, \mathrm{H}-1_{\mathrm{C}}\right)$, $4.55\left(\mathrm{~d}, 1 \mathrm{H}, J_{1,2} 7.8 \mathrm{~Hz}, \mathrm{H}-1_{\mathrm{B}}\right), 1.35(\mathrm{~d}, 3 \mathrm{H}$, $J \quad 4.4 \mathrm{~Hz}, \mathrm{CH}_{3} \mathrm{CH}$ ). Anal. Calcd for $\mathrm{C}_{36} \mathrm{H}_{49} \mathrm{O}_{24} \mathrm{Cl}$ : C, 47.98; H, 5.48. Found: $\mathrm{C}$, 47.73; H, 5.31.

3,6-Di-O-(2,3,4,6-tetra-O-acetyl- $\beta$-D-glucopyranosyl)-1,2-O-(R,S)-ethylidene- $\alpha-\mathrm{D}-$ gluco pyranose (11).- As described for the preparation of 10, the rearrangement of $9(780 \mathrm{mg}$, $0.9 \mathrm{mmol})$ with TMSOTf (0.1 equiv) in dry $\mathrm{CH}_{2} \mathrm{Cl}_{2}$ at $0{ }^{\circ} \mathrm{C}$ afforded $11(\mathrm{R}, \mathrm{S})$ in $76 \%$ yield (592 mg). Further purification by HPLC (1:1 petroleum ether-EtOAc) gave the $\mathrm{R}$ and $\mathrm{S}$ isomers as crystals. For $\mathrm{R}$ isomer: $\mathrm{mp}$ 87$88^{\circ} \mathrm{C} ;[\alpha]_{\mathrm{D}}^{20}-2.3^{\circ}\left(c 0.8, \mathrm{CHCl}_{3}\right) ;{ }^{1} \mathrm{H}$ NMR: $\delta$ $5.56\left(\mathrm{~d}, 1 \mathrm{H}, J_{1,2} 5.0 \mathrm{~Hz}, \mathrm{H}-1_{\mathrm{D}}\right), 5.21(\mathrm{q}, 1 \mathrm{H}$, $\left.J 5.2 \mathrm{~Hz}, \mathrm{CH}_{3} \mathrm{CH}\right), 4.90\left(\mathrm{~d}, 1 \mathrm{H}, J_{1,2} 8.0 \mathrm{~Hz}\right.$, $\left.\mathrm{H}-1_{\mathrm{F}}\right), 4.73\left(\mathrm{~d}, 1 \mathrm{H}, J_{1,2} 8.0 \mathrm{~Hz}, \mathrm{H}-1_{\mathrm{E}}\right), 2.15-$ $2.00\left(8 \mathrm{~s}, 24 \mathrm{H}, 8 \mathrm{CH}_{3} \mathrm{CO}\right), 1.48(\mathrm{~d}, 3 \mathrm{H}, J 5.2$ $\mathrm{Hz}, \mathrm{CH}_{3} \mathrm{CH}$ ); For $\mathrm{S}$ isomer: $\mathrm{mp} 77-78^{\circ} \mathrm{C}$; $[\alpha]_{\mathrm{D}}^{20}+1.7^{\circ}\left(c 0.4, \mathrm{CHCl}_{3}\right) ;{ }^{1} \mathrm{H}$ NMR: $\delta 5.63$ $\left(\mathrm{q}, 1 \mathrm{H}, J 5.5 \mathrm{~Hz}, \mathrm{CH}_{3} \mathrm{CH}\right), 5.51\left(\mathrm{~d}, 1 \mathrm{H}, J_{1,2}\right.$ $\left.5.1 \mathrm{~Hz}, \mathrm{H}-1_{\mathrm{D}}\right), 4.85\left(\mathrm{~d}, 1 \mathrm{H}, J_{1,2} 8.2 \mathrm{~Hz}, \mathrm{H}-1_{\mathrm{F}}\right)$, $4.72\left(\mathrm{~d}, 1 \mathrm{H}, J_{1.2} 8.2 \mathrm{~Hz}, \mathrm{H}-1_{\mathrm{E}}\right), 1.32(\mathrm{~d}, 3 \mathrm{H}$, $\left.J 5.5 \mathrm{~Hz}, \mathrm{CH}_{3} \mathrm{CH}\right)$. Anal. Calcd for $\mathrm{C}_{36} \mathrm{H}_{50} \mathrm{O}_{24}$ : C, 49.89; H, 5.81. Found: C, 49.76; H, 5.92. 
4-O-Acetyl-3-O-(2,3,4,6-tetra-O-acetyl- $\beta$-Dglucopyranosyl)-6-O-(2,3,4-tri-O-acetyl-6-Ochloroacetyl- $\beta$-D-glucopyranosyl)-1,2-O-(R,S)ethylidene- $\alpha$-D-glucopyranose (12)

Method $A$. To a stirred solution of $\mathbf{1 0}(480$ $\mathrm{mg})$ in pyridine $(1 \mathrm{~mL})$ was added $\mathrm{Ac}_{2} \mathrm{O}(0.5$ $\mathrm{mL})$ dropwise at $0{ }^{\circ} \mathrm{C}$, and the reaction was continued for $2 \mathrm{~h}$. The reaction mixture was poured into ice-water and extracted with $\mathrm{CH}_{2} \mathrm{Cl}_{2}(3 \times 5 \mathrm{~mL})$. The organic layer was washed with $\mathrm{N} \mathrm{HCl}(10 \mathrm{~mL})$, satd aq $\mathrm{Na}_{2} \mathrm{CO}_{3}$ $(10 \mathrm{~mL})$ and $\mathrm{H}_{2} \mathrm{O}(10 \mathrm{~mL})$, dried, and concentrated. The residue was purified by column chromatography (1:1 petroleum ether-EtOAc) to furnish $12(\mathrm{R}, \mathrm{S})(450 \mathrm{mg}, 90 \%)$.

Method B. To a mixture of $7(580 \mathrm{mg}, 1.0$ mmol), 6- $O$-chloroacetylated 'acetobromoglucose' (504 mg, $1.2 \mathrm{mmol}), 2$,4-lutidine $(80 \mu \mathrm{L}$, $0.69 \mathrm{mmol})$, and $4 \AA$ molecular sieves $(0.5 \mathrm{~g})$ in $\mathrm{CH}_{2} \mathrm{Cl}_{2}$ (dry, $20 \mathrm{~mL}$ ) was added silver triflate (308 mg, $1.2 \mathrm{mmol}$ ) under a nitrogen atmosphere in a dark room at $-5^{\circ} \mathrm{C}$. The reaction was carried out at $-5^{\circ} \mathrm{C}$ and monitored by TLC (1:1 petroleum ether-EtOAc). After completion of the reaction, pyridine $(3 \mathrm{~mL})$ and $10 \%$ aq $\mathrm{Na}_{2} \mathrm{~S}_{2} \mathrm{O}_{3}(20 \mathrm{~mL})$ were added and to the mixture, the mixture was filtered, and the solid was washed with $\mathrm{CH}_{2} \mathrm{Cl}_{2}(20 \mathrm{~mL})$. The filtrate was treated as for the preparation of 3 to furnish $12(\mathrm{R}, \mathrm{S})$ in $80 \%$ yield $(750 \mathrm{mg})$. Further purification by HPLC (1:1 petroleum ether-EtOAc) gave the $\mathrm{R}$ and $\mathrm{S}$ isomers as syrups. For $\mathrm{R}$ isomer: $[\alpha]_{\mathrm{D}}^{20}-11.2^{\circ}(c) 0.5$, $\left.\mathrm{CHCl}_{3}\right)$; ${ }^{1} \mathrm{H}$ NMR: $\delta 5.52\left(\mathrm{~d}, 1 \mathrm{H}, J_{1,2} 4.8 \mathrm{~Hz}\right.$, $\left.\mathrm{H}-1_{\mathrm{A}}\right), 5.30-4.88\left(\mathrm{~m}, 8 \mathrm{H}, \mathrm{H}-2_{\mathrm{B}}, 2_{\mathrm{C}}, 3_{\mathrm{B}}, 3_{\mathrm{C}}, 4_{\mathrm{A}}\right.$, $\left.4_{\mathrm{B}}, 4_{\mathrm{C}}, \mathrm{CH}_{3} \mathrm{CH}\right), 4.80\left(\mathrm{~d}, 1 \mathrm{H}, J_{1,2} 8.0 \mathrm{~Hz}\right.$, $\left.\mathrm{H}-1_{\mathrm{C}}\right), 4.66\left(\mathrm{~d}, 1 \mathrm{H}, J_{1,2} 8.0 \mathrm{~Hz}, \mathrm{H}-1_{\mathrm{B}}\right), 4.44-$ $3.56\left(\mathrm{~m}, 11 \mathrm{H}, \mathrm{H}-2_{\mathrm{A}}, 3_{\mathrm{A}}, 5_{\mathrm{A}}, 5_{\mathrm{B}}, 5_{\mathrm{C}}, 6_{\mathrm{A}}, 6_{\mathrm{B}}, 6_{\mathrm{C}}\right)$, 4.11 (s, $\left.2 \mathrm{H}, \mathrm{CH}_{2} \mathrm{ClCO}\right), 2.08,2.07,2.07,2.03$, 2.02, 2.01, 2.01, 1.99 (8 s, $\left.24 \mathrm{H}, 8 \mathrm{CH}_{3} \mathrm{CO}\right)$, $1.43\left(\mathrm{~d}, 3 \mathrm{H}, \mathrm{J} 4.8 \mathrm{~Hz}, \mathrm{CH}_{3} \mathrm{CH}\right)$; For $\mathrm{S}$ isomer: $[\alpha]_{\mathrm{D}}^{20}+5.5^{\circ}\left(c 0.7, \mathrm{CHCl}_{3}\right) ;{ }^{1} \mathrm{H} \mathrm{NMR}: \delta 5.61(\mathrm{~d}$, $\left.1 \mathrm{H}, J_{1,2} 4.5 \mathrm{~Hz}, \mathrm{H}-1_{\mathrm{A}}\right), 5.49$ (q, $1 \mathrm{H}, J 4.4 \mathrm{~Hz}$, $\left.\mathrm{CH}_{3} \mathrm{CH}\right), 4.77$ (d, $\left.1 \mathrm{H}, J_{1,2} 8.0 \mathrm{~Hz}, \mathrm{H}-1_{\mathrm{C}}\right), 4.53$ $\left(\mathrm{d}, 1 \mathrm{H}, J_{1,2} 7.9 \mathrm{~Hz}, \mathrm{H}-1_{\mathrm{B}}\right), 1.35$ (d, $3 \mathrm{H}, J 4.4$ $\mathrm{Hz}, \mathrm{CH}_{3} \mathrm{CH}$ ). Anal. Calcd for $\mathrm{C}_{38} \mathrm{H}_{51} \mathrm{O}_{25} \mathrm{Cl}$ : C, 48.39; H, 5.45. Found: C, 48.16; H, 5.31.

4-O-Acetyl-3,6-di-O-(2,3,4,6-tetra-O-acetyl$\beta$-D-glucopyranosyl)-1,2-O-( R,S)-ethylidene- $\alpha$ D-glucopyranose (13)

Method A. Acetylation of 11 (480 mg) with
$\mathrm{Ac}_{2} \mathrm{O} /$ pyridine at $\mathrm{rt}$ for $2 \mathrm{~h}$ furnished $\mathbf{1 3}$ (500 $\mathrm{mg}$ ) in quantitative yield.

Method $B$. Under the same conditions as described for the preparation of $\mathbf{1 2}$, the coupling of 7 (580 mg, $1 \mathrm{mmol})$ and 'acetobromoglucose' (492 mg, $1.2 \mathrm{mmol}$ ) furnished $\mathbf{1 3}$ $(\mathrm{R}, \mathrm{S})$ as an amorphous solid in $85 \%$ yield (770 mg). Further purification by HPLC (1:1 petroleum ether-EtOAc) gave the $\mathrm{R}$ and $\mathrm{S}$ isomers as syrups. For $\mathrm{R}$ isomer: $[\alpha]_{\mathrm{D}}^{20}-0.9^{\circ}$ (c $\left.0.5, \mathrm{CHCl}_{3}\right) ;{ }^{1} \mathrm{H} \mathrm{NMR}: \delta 5.50\left(\mathrm{~d}, 1 \mathrm{H}, J_{1,2}\right.$ $\left.4.8 \mathrm{~Hz}, \mathrm{H}-1_{\mathrm{D}}\right), 5.25-5.15\left(\mathrm{~m}, 2 \mathrm{H}, \mathrm{H}-4_{\mathrm{D}}\right.$, $\left.\mathrm{CH}_{3} \mathrm{CH}\right), 5.12-4.93\left(\mathrm{~m}, 6 \mathrm{H}, \mathrm{H}-2_{\mathrm{E}}, 2_{\mathrm{F}}, 3_{\mathrm{E}}\right.$, $\left.3_{\mathrm{F}}, 4_{\mathrm{E}}, 4_{\mathrm{F}}\right), 4.80\left(\mathrm{~d}, 1 \mathrm{H}, J_{1,2} 8.2 \mathrm{~Hz}, \mathrm{H}-1_{\mathrm{F}}\right)$, $4.63\left(\mathrm{~d}, 1 \mathrm{H}, J_{1,2} 8.2 \mathrm{~Hz}, \mathrm{H}-1_{\mathrm{E}}\right), 4.28-3.60$ $\left(\mathrm{m}, 12 \mathrm{H}, \mathrm{H}-2_{\mathrm{D}}, 3_{\mathrm{D}}, 4_{\mathrm{D}}, 5_{\mathrm{D}}, 5_{\mathrm{E}}, 5_{\mathrm{F}}, 6_{\mathrm{D}}, 6_{\mathrm{E}}\right.$, $\left.6_{\mathrm{F}}\right), 2.08,2.07,2.07,2.06,2.04,2.02,2.02$, 2.00, $2.00\left(9 \mathrm{~s}, 27 \mathrm{H}, 9 \mathrm{CH}_{3} \mathrm{CO}\right), 1.45$ (d, 3 $\mathrm{H}, J 4.8 \mathrm{~Hz}, \mathrm{CH}_{3} \mathrm{CH}$ ); For $\mathrm{S}$ isomer: $[\alpha]_{\mathrm{D}}^{20}+$ $27.5^{\circ}\left(c 0.3, \mathrm{CHCl}_{3}\right)$; ${ }^{1} \mathrm{H}$ NMR: $\delta 5.63(\mathrm{~d}, 1$ $\left.\mathrm{H}, J_{1,2} 4.4 \mathrm{~Hz}, \mathrm{H}-1_{\mathrm{D}}\right), 5.50(\mathrm{q}, 1 \mathrm{H}, J 4.7 \mathrm{~Hz}$, $\left.\mathrm{CH}_{3} \mathrm{CH}\right), 4.81\left(\mathrm{~d}, 1 \mathrm{H}, J_{1,2} 7.9 \mathrm{~Hz}, \mathrm{H}-1_{\mathrm{F}}\right)$, $4.54\left(\mathrm{~d}, 1 \mathrm{H}, J_{1,2} 7.9 \mathrm{~Hz}, \mathrm{H}-1_{\mathrm{E}}\right), 1.36(\mathrm{~d}, 3 \mathrm{H}$, J $\left.4.7 \mathrm{~Hz}, \quad \mathrm{C} \mathrm{H}_{3} \mathrm{CH}\right)$. Anal. Calcd for $\mathrm{C}_{38} \mathrm{H}_{52} \mathrm{O}_{25}$ : C, 50.22; H, 5.77. Found: C, $50.48 ; \mathrm{H}, 5.58$.

3,6-Di-O-(2,3,4,6-tetra-O-acetyl- $\beta$-D-glucopyranosyl)-1,2,4-tri-O-acetyl- $\alpha, \beta$-D-glucopyranose (15)._Compound 13 (481 mg, 0.53 mmol) was treated with $90 \% \quad \mathrm{~F}_{3} \mathrm{CCOOH}$ (3 $\mathrm{mL}$ ) at $\mathrm{rt}$ for $1 \mathrm{~h}$, and the solution was concentrated and co-concentrated with toluene. The residue was dissolved in pyridine $(5 \mathrm{~mL})$ and treated with $\mathrm{Ac}_{2} \mathrm{O}$ (3 $\mathrm{mL}$ ) for $2 \mathrm{~h}$. After conventional work-up, the residue was subjected to column chromatography (1:1 petroleum ether-EtOAc) to yield the title compound $\mathbf{1 5}$ as a syrupy mixture of $\alpha$ and $\beta$ anomers in a $2: 1$ ratio $(358 \mathrm{mg}$, $70 \%$ ); $[\alpha]_{\mathrm{D}}^{20}-1.9^{\circ}\left(\right.$ c $\left.1.1, \mathrm{CHCl}_{3}\right) ;{ }^{1} \mathrm{H}$ NMR: $\delta$ $6.21\left(\mathrm{~d}, 2 / 3 \mathrm{H}, J_{1,2} 3.8 \mathrm{~Hz}, \mathrm{H}-1_{\mathrm{D} \alpha}\right), 5.59(\mathrm{~d}$, $\left.1 / 3 \mathrm{H}, J_{1,2} 7.8 \mathrm{~Hz}, \mathrm{H}-1_{\mathrm{D} \beta}\right), 5.21-4.80(\mathrm{~m}, 7$ $\left.\mathrm{H}, \mathrm{H}-2_{\mathrm{D}}, 2_{\mathrm{E}}, 2_{\mathrm{F}}, 3_{\mathrm{E}}, 3_{\mathrm{F}}, 4_{\mathrm{E}}, 4_{\mathrm{F}}\right), 4.65(\mathrm{~d}, 2 / 3$ $\left.\mathrm{H}, J_{1,2} 8.4 \mathrm{~Hz}, \mathrm{H}-1_{\mathrm{F} \alpha}\right), 4.60\left(\mathrm{~d}, 1 / 3 \mathrm{H}, J_{1,2} 8.4\right.$ $\left.\mathrm{Hz}, \mathrm{H}-1_{\mathrm{F} \beta}\right), 4.56$ (d, 1/3 H, J ${ }_{1,2} 8.4 \mathrm{~Hz}, \mathrm{H}-$ $\left.1_{\mathrm{E} \beta}\right), 4.50\left(\mathrm{~d}, 2 / 3 \mathrm{H}, J_{1,2} 8.4 \mathrm{~Hz}, \mathrm{H}-1_{\mathrm{E} \alpha}\right)$, $4.46-3.40\left(\mathrm{~m}, 11 \mathrm{H}, \mathrm{H}-3_{\mathrm{D}}, 4_{\mathrm{D}}, 5_{\mathrm{D}}, 5_{\mathrm{E}}, 5_{\mathrm{F}}, 6_{\mathrm{D}}\right.$, $\left.6_{\mathrm{E}}, 6_{\mathrm{F}}\right), 2.19-1.97\left(\mathrm{~m}, 33 \mathrm{H}, 11 \mathrm{CH}_{3} \mathrm{CO}\right)$. Anal. Calcd for $\mathrm{C}_{40} \mathrm{H}_{54} \mathrm{O}_{27}$ : C, 49.69; H, 5.63. Found: C, 49.44; H, 5.78. 
2,4-Di-O-acetyl-3,6-di-O-(2,3,4,6-tetra-Oacetyl- $\beta$-D-glucopyranosyl)- $\alpha, \beta$-D-glucopyranose (16). - To a stirred solution of $15(242 \mathrm{mg}$, $0.25 \mathrm{mmol}$ ) in DMF (dry, $1 \mathrm{~mL}$ ) was added hydrazine acetate $(24 \mathrm{mg}, 0.26 \mathrm{mmol})$. Stirring was maintained at $\mathrm{rt}$ for $50 \mathrm{~min}$. The mixture was poured into $\mathrm{H}_{2} \mathrm{O}(10 \mathrm{~mL})$ and extracted with $\mathrm{CH}_{2} \mathrm{Cl}_{2}(3 \times 10 \mathrm{~mL})$. Combined extracts were washed with $\mathrm{H}_{2} \mathrm{O}(3 \times 10 \mathrm{~mL})$, dried, and concentrated to give $16(226 \mathrm{mg}, 98 \%)$ as a syrupy anomeric mixture $(\alpha / \beta, 1: 3)$ after column chromatography $(1: 1$ petroleum ether-EtOAc); $[\alpha]_{\mathrm{D}}^{20}+3.8^{\circ}\left(c 1.1, \mathrm{CHCl}_{3}\right) ;{ }^{1} \mathrm{H}$ NMR: $\delta 5.37\left(\mathrm{~d}, 1 / 4 \mathrm{H}, J_{1,2} 3.8 \mathrm{~Hz}, \mathrm{H}-1_{\mathrm{D} \alpha}\right)$, $5.35\left(\mathrm{~d}, 3 / 4 \mathrm{H}, J_{1,2} 3.6 \mathrm{~Hz}, \mathrm{H}-1_{\mathrm{D} \beta}\right), 5.22(\mathrm{t}, 1$ $\left.\mathrm{H}, J 9.6 \mathrm{~Hz}, \mathrm{H}-4_{\mathrm{D}}\right), 5.13-4.78\left(\mathrm{~m}, 7 \mathrm{H}, \mathrm{H}-2_{\mathrm{D}}\right.$, $\left.2_{\mathrm{E}}, 2_{\mathrm{F}}, 3_{\mathrm{E}}, 3_{\mathrm{F}}, 4_{\mathrm{E}}, 4_{\mathrm{F}}\right), 4.67\left(\mathrm{~d}, 1 \mathrm{H}, J_{1,2} 8.1 \mathrm{~Hz}\right.$, $\left.\mathrm{H}-1_{\mathrm{F}}\right), 4.56\left(\mathrm{~d}, 1 \mathrm{H}, J_{1,2} 7.8 \mathrm{~Hz}, \mathrm{H}-1_{\mathrm{E}}\right), 4.34-$ $3.58\left(\mathrm{~m}, 10 \mathrm{H}, \mathrm{H}-3_{\mathrm{D}}, 5_{\mathrm{D}}, 5_{\mathrm{E}}, 5_{\mathrm{F}}, 6_{\mathrm{D}}, 6_{\mathrm{E}}, 6_{\mathrm{F}}\right)$, 2.10-1.98 (m, 30 H, $\left.10 \mathrm{CH}_{3} \mathrm{CO}\right)$. Anal. Calcd for $\mathrm{C}_{38} \mathrm{H}_{52} \mathrm{O}_{26}$ : C, 49.35; H, 5.67. Found: C, 49.12; H, 5.78 .

2,4-Di-O-acetyl-3,6-di-O-(2,3,4,6-tetra-Oacetyl- $\beta$-D-glucopyranosyl)- $\alpha-\mathrm{D}-$ glucopyranos$y l$ trichloroacetimidate (17). - Trichloroacetonitrile $\left(\mathrm{Cl}_{3} \mathrm{CCN}, 69 \mu \mathrm{L}, 3\right.$ equiv) and 1,8-diazabicyclo[5,4,0]undec-7-ene (DBU, $9 \mu \mathrm{L}, 0.25$ equiv) were added to a solution of $16(203 \mathrm{mg}$, $0.22 \mathrm{mmol})$ in anhyd $\mathrm{CH}_{2} \mathrm{Cl}_{2}(10 \mathrm{~mL})$, and the mixture was stirred at $0{ }^{\circ} \mathrm{C}$ for $1 \mathrm{~h}$, at the end of which time TLC showed the starting material had disappeared. The solvents were evaporated, and flash chromatography (1:1 EtOAc-petroleum ether) of the residue gave the trisaccharide donor 17 (188 $\mathrm{mg}, 80 \%)$, which was used directly in the glycosylation reaction. ${ }^{1} \mathrm{H} \mathrm{NMR}\left(\mathrm{CDCl}_{3}\right): \delta 8.50(\mathrm{bs}, 1 \mathrm{H}$, $\mathrm{NH}), 6.53$ (bs, $\left.1 \mathrm{H}, \mathrm{H}-1_{\mathrm{D}}\right), 5.30$ (t, $1 \mathrm{H}, J 9.6$ $\left.\mathrm{Hz}, \mathrm{H}-4_{\mathrm{D}}\right), 5.26-4.67\left(\mathrm{~m}, 7 \mathrm{H}, \mathrm{H}-2_{\mathrm{D}}, 2_{\mathrm{E}}, 2_{\mathrm{F}}\right.$, $\left.3_{\mathrm{E}}, 3_{\mathrm{F}}, 4_{\mathrm{E}}, 4_{\mathrm{F}}\right), 4.69\left(\mathrm{~d}, 1 \mathrm{H}, J_{1,2} 8.1 \mathrm{~Hz}, \mathrm{H}-1_{\mathrm{F}}\right)$, $4.55\left(\mathrm{~d}, 1 \mathrm{H}, J_{1,2} 8.0 \mathrm{~Hz}, \mathrm{H}-1_{\mathrm{E}}\right), 4.50-3.48(\mathrm{~m}$, $\left.10 \mathrm{H}, \mathrm{H}-3_{\mathrm{D}}, 5_{\mathrm{D}}, 5_{\mathrm{E}}, 5_{\mathrm{F}}, 6_{\mathrm{D}}, 6_{\mathrm{E}}, 6_{\mathrm{F}}\right), 2.09,2.09$, $2.07,2.06,2.06,2.05,2.02,2.01,2.01,1.99$ (10 $\left.\mathrm{s}, 30 \mathrm{H}, 10 \mathrm{CH}_{3} \mathrm{CO}\right)$.

4-O-Acetyl-3-O-(2,3,4,6-tetra-O-acetyl- $\beta$-Dglucopyranosyl)-6-O-(2,3,4-tri-O-acetyl- $\beta$-Dglucopyranosyl)-1,2-O-(R,S)-ethylidene - $\alpha-\mathrm{D}$ glucopyranose (18). - Compound $12(280 \mathrm{mg}$, $0.30 \mathrm{mmol})$ was dissolved in anhyd EtOH (20 $\mathrm{mL}$ ), and thiourea (40 $\mathrm{mg}$ ) was added. The mixture was heated at $90^{\circ} \mathrm{C}$ for $1 \mathrm{~h}$, at the end of which time TLC showed the starting material had disappeared. The reaction was cooled to $\mathrm{rt}$, and the mixture extracted with $\mathrm{CH}_{2} \mathrm{Cl}_{2}(20 \mathrm{~mL})$. The organic solution was washed with $\mathrm{H}_{2} \mathrm{O}(20 \mathrm{~mL})$ and concentrated, and the residue was subjected to column chromatography (1:1 petroleum ether-EtOAc) to yield the title compound 18 (193 mg, 74\%). Further purification by HPLC (1:1 petroleum ether-EtOAc) gave the $\mathrm{R}$ and $\mathrm{S}$ isomers as crystals. For $\mathrm{R}$ isomer: $\mathrm{mp} 93-95^{\circ} \mathrm{C} ;[\alpha]_{\mathrm{D}}^{20}-$ $1.3^{\circ}\left(c 1.0, \mathrm{CHCl}_{3}\right) ;{ }^{1} \mathrm{H} \mathrm{NMR}: \delta 5.51(\mathrm{~d}, 1 \mathrm{H}$, $\left.J_{1,2} 4.7 \mathrm{~Hz}, \mathrm{H}-1_{\mathrm{A}}\right), 5.22-4.90\left(\mathrm{~m}, 8 \mathrm{H}, \mathrm{H}-2_{\mathrm{B}}\right.$, $\left.2_{\mathrm{C}}, 3_{\mathrm{B}}, 3_{\mathrm{C}}, 4_{\mathrm{A}}, 4_{\mathrm{B}}, 4_{\mathrm{C}}, \mathrm{CH}_{3} \mathrm{CH}\right), 4.76(\mathrm{~d}, 1 \mathrm{H}$, $\left.J_{1,2} 8.0 \mathrm{~Hz}, \mathrm{H}-1_{\mathrm{C}}\right), 4.58\left(\mathrm{~d}, 1 \mathrm{H}, J_{1,2} 8.0 \mathrm{~Hz}\right.$, $\left.\mathrm{H}-1_{\mathrm{B}}\right), 4.21-3.45\left(\mathrm{~m}, 11 \mathrm{H}, \mathrm{H}-2_{\mathrm{A}}, 3_{\mathrm{A}}, 5_{\mathrm{A}}, 5_{\mathrm{B}}\right.$, $\left.5_{\mathrm{C}}, 6_{\mathrm{A}}, 6_{\mathrm{B}}, 6_{\mathrm{C}}\right), 2.09,2.07,2.05,2.03,2.00$, $1.96,1.93,1.91\left(8 \mathrm{~s}, 8 \mathrm{CH}_{3} \mathrm{CO}\right), 1.41(\mathrm{~d}, 3 \mathrm{H}$, $J 5.1 \mathrm{~Hz}, \mathrm{CH}_{3} \mathrm{CH}$ ); For $\mathrm{S}$ isomer: $\mathrm{mp} 87-$ $\left.90{ }^{\circ} \mathrm{C} ;[\alpha]_{\mathrm{D}}^{20}+12.6^{\circ}(c) 1.4, \mathrm{CHCl}_{3}\right) ;{ }^{1} \mathrm{H} \mathrm{NMR}$ : $\delta 5.55\left(\mathrm{~d}, 1 \mathrm{H}, J_{1,2} 4.6 \mathrm{~Hz}, \mathrm{H}-1_{\mathrm{A}}\right), 5.46(\mathrm{q}, 1 \mathrm{H}$, $\left.J 4.9 \mathrm{~Hz}, \mathrm{CH}_{3} \mathrm{CH}\right), 4.72\left(\mathrm{~d}, 1 \mathrm{H}, J_{1,2} 8.0 \mathrm{~Hz}\right.$, $\left.\mathrm{H}-1_{\mathrm{C}}\right), 4.51\left(\mathrm{~d}, 1 \mathrm{H}, J_{1,2} 8.0 \mathrm{~Hz}, \mathrm{H}-1_{\mathrm{B}}\right), 1.28$ (d, $3 \mathrm{H}, J$ $\left.4.9 \mathrm{~Hz}, \mathrm{CH}_{3} \mathrm{CH}\right)$. Anal. Calcd for $\mathrm{C}_{36} \mathrm{H}_{50} \mathrm{O}_{24}: \mathrm{C}$, 49.89; $\mathrm{H}$, 5.81. Found: $\mathrm{C}$, 49.75; $\mathrm{H}, 5.81$.

4-O-Acetyl-3-O-(2,3,4,6-tetra-O-acetyl- $\beta$-Dglucopyranosyl)-6-O- (2,3,4-tri-O-acetyl-6-O(2,4-di-O - acetyl-3,6-di-O - (2,3,4,6-tetra-Oacetyl- $\beta$-D-glucopyranosyl)- $\beta$-D-glucopyranosyl)- $\beta$ - D-glucopyranosyl)- 1,2-O-(R,S)-ethylidene- $\alpha$-D-glucopyranose (19). - A mixture of compound 17 (50 mg, $0.047 \mathrm{mmol})$ and 18 (41 $\mathrm{mg}, 0.047 \mathrm{mmol})$ in anhyd $\mathrm{CH}_{2} \mathrm{Cl}_{2}(10 \mathrm{~mL})$ under $\mathrm{N}_{2}$ was stirred with activated $4 \AA$ molecular sieves for $1 \mathrm{~h}$ at $\mathrm{rt}$. The mixture was cooled to $-78^{\circ} \mathrm{C}$ and TMSOTf $(0.8 \mu \mathrm{L}, 0.1$ equiv) in anhyd $\mathrm{CH}_{2} \mathrm{Cl}_{2}(1 \mathrm{~mL})$ was added dropwise. The mixture was stirred at $-30{ }^{\circ} \mathrm{C}$ for $30 \mathrm{~min}$, at the end of which time TLC $(1: 1.5$ petroleum ether-EtOAc) indicated that the reaction was complete. The reaction mixture was neutralized with $\mathrm{Et}_{3} \mathrm{~N}(0.1 \mathrm{~mL})$, filtered through Celite, and the filtrate was concentrated. The residue was purified by column chromatography with $1: 1.5$ petroleum ether-EtOAc as the eluent to yield $19(\mathrm{R}, \mathrm{S})$ as an amorphous solid (56 mg, 68\%). Further purification of $19(\mathrm{R}, \mathrm{S})$ by HPLC with $1: 1.5$ petroleum ether-EtOAc as the eluent furnished the $\mathrm{R}$ and $\mathrm{S}$ isomers. For $\mathrm{R}$ isomer: $\mathrm{mp}$ 
97-100 ${ }^{\circ} \mathrm{C},[\alpha]_{\mathrm{D}}^{20}-8.6^{\circ}\left(c \quad 0.5, \mathrm{CHCl}_{3}\right) ;{ }^{1} \mathrm{H}$ NMR: $\delta 5.51\left(\mathrm{~d}, 1 \mathrm{H}, J_{1,2} 4.1 \mathrm{~Hz}, \mathrm{H}-1_{\mathrm{A}}\right)$, $5.25-4.83\left(\mathrm{~m}, 16 \mathrm{H}, \mathrm{H}-2_{\mathrm{B}}, 2_{\mathrm{C}}, 2_{\mathrm{D}}, 2_{\mathrm{E}}, 2_{\mathrm{F}}, 3_{\mathrm{B}}\right.$, $\left.3_{\mathrm{C}}, 3_{\mathrm{E}}, 3_{\mathrm{F}}, 4_{\mathrm{A}}, 4_{\mathrm{B}}, 4_{\mathrm{C}}, 4_{\mathrm{D}}, 4_{\mathrm{E}}, 4_{\mathrm{F}}, \mathrm{CH}_{3} \mathrm{CH}\right)$, $4.80,4.73,4.62,4.58,4.56\left(5 \mathrm{~d}, 5 \mathrm{H}, J_{1,2} 8.2\right.$ $\left.\mathrm{Hz}, \mathrm{H}-1_{\mathrm{B}}, 1_{\mathrm{C}}, 1_{\mathrm{D}}, 1_{\mathrm{E}}, 1_{\mathrm{F}}\right), 4.33-3.41(\mathrm{~m}, 21 \mathrm{H}$, $\mathrm{H}-2_{\mathrm{A}}, 3_{\mathrm{A}}, 3_{\mathrm{C}}, 5_{\mathrm{A}}, 5_{\mathrm{B}}, 5_{\mathrm{C}}, 5_{\mathrm{D}}, 5_{\mathrm{E}}, 5_{\mathrm{F}}, 6_{\mathrm{A}}, 6_{\mathrm{B}}, 6_{\mathrm{C}}$, $\left.6_{\mathrm{D}}, 6_{\mathrm{E}}, 6_{\mathrm{F}}\right), 2.13-1.93\left(\mathrm{~m}, 54 \mathrm{H}, 18 \mathrm{CH}_{3} \mathrm{CO}\right)$, 1.47 (d, $\left.3 \mathrm{H}, J 4.9 \mathrm{~Hz}, \mathrm{CH}_{3} \mathrm{CH}\right)$; For $\mathrm{S}$ isomer: $\operatorname{mp~} 102-103{ }^{\circ} \mathrm{C} ;[\alpha]_{\mathrm{D}}^{20}+15.9^{\circ}\left(c 0.9, \mathrm{CHCl}_{3}\right)$; ${ }^{1} \mathrm{H}$ NMR: $\delta 5.54\left(\mathrm{~d}, 1 \mathrm{H}, J_{1,2} 4.6 \mathrm{~Hz}, \mathrm{H}-1_{\mathrm{A}}\right)$, 5.45 (q, $\left.1 \mathrm{H}, J 4.4 \mathrm{~Hz}, \mathrm{CH}_{3} \mathrm{CH}\right), 4.74,4.67$, $4.60,4.55,4.49\left(5 \mathrm{~d}, 5 \mathrm{H}, J_{1,2} 8.2 \mathrm{~Hz}, \mathrm{H}-1_{\mathrm{B}}, 1_{\mathrm{C}}\right.$, $\left.1_{\mathrm{D}}, 1_{\mathrm{E}}, 1_{\mathrm{F}}\right), 1.23\left(\mathrm{~d}, 3 \mathrm{H}, J 4.4 \mathrm{~Hz}, \mathrm{CH}_{3} \mathrm{CH}\right)$. Anal. Calcd for $\mathrm{C}_{74} \mathrm{H}_{100} \mathrm{O}_{49}$ : C, 50.11; H, 5.68. Found: C, 50.04; H, 5.76.

1,2,4- Tri-O - acetyl- 3- O - (2,3,4,6-tetra-Oacetyl- $\beta$-D-glucopyranosyl)- $6-\mathrm{O}-(2,3,4-$ tri-Oacetyl-6-O-(2,4-di-O-acetyl-3,6-di-O- $(2,3,4,6$ tetra-O-acetyl- $\beta$-D-glucopyranosyl)- $\beta$-D-glucopyranosyl)- $\beta$-D-glucopyranosyl)- $\alpha, \beta$-D-glucopyranose (21). - As described in the preparation of 15, compound 19 (42 $\mathrm{mg}, 0.023 \mathrm{mmol})$ was treated with $90 \% \mathrm{~F}_{3} \mathrm{CCOOH}(1.5 \mathrm{~mL})$ at $\mathrm{rt}$ for $1.5 \mathrm{~h}$ and the mixture was concentrated and co-concentrated with toluene. The residue was dissolved in pyridine $(1 \mathrm{~mL})$ and treated with $\mathrm{Ac}_{2} \mathrm{O}(0.5 \mathrm{~mL})$ for $2 \mathrm{~h}$. After conventional work-up, the residue was subjected to column chromatography $(1: 1.5$ petroleum ether-EtOAc) to yield the title compound 21 $(\alpha / \beta, 2: 1) \quad(30 \mathrm{mg}, 72 \%)$ as an amorphous solid; $\mathrm{mp} 108-111^{\circ} \mathrm{C} ;[\alpha]_{\mathrm{D}}^{20}+16.0^{\circ}$ (c) 0.5 , $\left.\mathrm{CHCl}_{3}\right) ;{ }^{1} \mathrm{H}$ NMR: $\delta 6.13\left(\mathrm{~d}, 2 / 3 \mathrm{H}, J_{1,2} 3.1\right.$ $\left.\mathrm{Hz}, \mathrm{H}-1_{\mathrm{A} \alpha}\right), 5.50\left(\mathrm{~d}, 1 / 3 \mathrm{H}, J_{1,2} 9.3 \mathrm{~Hz}, \mathrm{H}-1_{\mathrm{A} \beta}\right)$, $5.47-5.13\left(\mathrm{~m}, 16 \mathrm{H}, \mathrm{H}-2_{\mathrm{A}}, 2_{\mathrm{B}}, 2_{\mathrm{C}}, 2_{\mathrm{D}}, 2_{\mathrm{E}}, 2_{\mathrm{F}}\right.$, $\left.3_{\mathrm{B}}, 3_{\mathrm{C}}, 3_{\mathrm{E}}, 3_{\mathrm{F}}, 4_{\mathrm{A}}, 4_{\mathrm{B}}, 4_{\mathrm{C}}, 4_{\mathrm{D}}, 4_{\mathrm{E}}, 4_{\mathrm{F}}\right), 4.85-4.45$ $\left(\mathrm{m}, 5 \mathrm{H}, J_{1,2} 8.3 \mathrm{~Hz}, \mathrm{H}-1_{\mathrm{B}}, 1_{\mathrm{C}}, 1_{\mathrm{D}}, 1_{\mathrm{E}}, 1_{\mathrm{F}}\right)$, $4.40-3.35\left(\mathrm{~m}, 20 \mathrm{H}, \mathrm{H}-3_{\mathrm{A}}, 3_{\mathrm{D}}, 5_{\mathrm{A}}, 5_{\mathrm{B}}, 5_{\mathrm{C}}, 5_{\mathrm{D}}\right.$, $\left.5_{\mathrm{E}}, 5_{\mathrm{F}}, 6_{\mathrm{A}}, 6_{\mathrm{B}}, 6_{\mathrm{C}}, 6_{\mathrm{D}}, 6_{\mathrm{E}}, 6_{\mathrm{F}}\right), 2.04-1.88(\mathrm{~m}, 60$ $\left.\mathrm{H}, 20 \mathrm{CH}_{3} \mathrm{CO}\right)$. Anal. Calcd for $\mathrm{C}_{76} \mathrm{H}_{102} \mathrm{O}_{51}$ : C, 49.84; H, 5.61. Found: C, 49.66; H, 5.51.

3,4,6-Tri-O-(2,3,4,6-tetra-O-acetyl- $\beta$-D-glucopyranosyl) - 1,2 - O - (R,S) -ethylidene - $\alpha$ - Dglucopyranose (22). - A solution of acetobromoglucose (410 g, $1.0 \mathrm{mmol}), 4$ (540 mg, 1.0 mmol), and $4 \AA$ activated molecular sieves (1 g) in anhyd $\mathrm{CH}_{2} \mathrm{Cl}_{2}(20 \mathrm{~mL})$ was stirred under $\mathrm{N}_{2}$ for $1 \mathrm{~h}$ at $\mathrm{rt}$ and then cooled to $-5^{\circ} \mathrm{C}$. A solution of silver triflate $(260 \mathrm{mg}, 1.0 \mathrm{mmol})$ and 2 ,4-lutidine $(0.1 \mathrm{~mL}, 0.87 \mathrm{mmol})$ in $1: 1$ anhyd $\mathrm{CH}_{2} \mathrm{Cl}_{2}$-toluene $(5 \mathrm{~mL})$ was added dropwise with stirring, over a $30 \mathrm{~min}$ period under $\mathrm{N}_{2}$. The mixture was stirred for $4 \mathrm{~h}$, at the end of which time TLC (2:1 petroleum ether-EtOAc) showed the presence of one major product and the disappearance of acetobromoglucose. The reaction was quenched with $0.4 \mathrm{~mL}$ pyridine and $4 \mathrm{~mL} 10 \%$ aq $\mathrm{Na}_{2} \mathrm{~S}_{2} \mathrm{O}_{3}$, the mixture was filtered through Celite, and the filtrate was handled as described for the preparation of $\mathbf{3}$ to yield the tetrasaccharide 22 (R,S) (361 mg, 30\% based on 4). After purification by column chromatography (1:1 petroleum ether-EtOAc) and by analytical LC (2:1 petroleum etherEtOAc), the pure $\mathrm{R}$ and $\mathrm{S}$ isomers were obtained; ${ }^{1} \mathrm{H}$ NMR $(\mathrm{R}): \delta 5.40(\mathrm{~d}, 1 \mathrm{H}, J 5.1 \mathrm{~Hz}$, $\mathrm{H}-1$ ), 5.28-4.93 (m, $10 \mathrm{H}, \mathrm{H}-2^{\prime}, 2^{\prime \prime}, 2^{\prime \prime \prime}, 3^{\prime}, 3^{\prime \prime}$, $\left.3^{\prime \prime \prime}, 4^{\prime}, 4^{\prime \prime}, 4^{\prime \prime \prime}, \mathrm{CH}_{3} \mathrm{CH}\right), 4.84,4.70,4.68$ (3 d, $\left.3 \mathrm{H}, J 7.9 \mathrm{~Hz}, \mathrm{H}-1^{\prime}, 1^{\prime \prime}, 1^{\prime \prime \prime}\right), 4.44-3.70$ (m, 15 H, H-2, 3, 4, 5, 5', 5", 5'", 6, 6', 6", 6"' $), 2.08$, $2.07,2.05,2.04,2.04,2.04,2.03,2.03,2.03$, 2.02, 2.01, $2.01\left(12 \mathrm{~s}, 36 \mathrm{H}, \mathrm{CH}_{3} \mathrm{CO}\right), 1.46(\mathrm{~d}$, $\left.3 \mathrm{H}, J 4.8 \mathrm{~Hz}, \mathrm{CH}_{3} \mathrm{CH}\right)$; (S): $\delta 5.56(\mathrm{q}, 1 \mathrm{H}, J$ $\left.4.1 \mathrm{~Hz}, \mathrm{CH}_{3} \mathrm{CH}\right), 5.44(\mathrm{~d}, 1 \mathrm{H}, J 5.1 \mathrm{~Hz}, \mathrm{H}-1)$, 4.80, 4.65, 4.64 (3 d, $3 \mathrm{H}, J 8.2 \mathrm{~Hz}, \mathrm{H}-1^{\prime}, 1^{\prime \prime}$, $\left.1^{\prime \prime \prime}\right), 1.37\left(\mathrm{~d}, 3 \mathrm{H}, J 4.1 \mathrm{~Hz}, \mathrm{CH}_{3} \mathrm{CH}\right)$.

3-O-( $\beta$-D-Glucopyranosyl)- 6-O-(6-O- $(3,6-$ di-O-( $\beta$ - D-glucopyranosyl)- $\beta$ - D-glucopyranosyl)- $\beta$-D-glucopyranosyl)- $\alpha, \beta$-D-glucopyranose (1). - The protected hexasaccharide 21 (19 $\mathrm{mg}, 0.01 \mathrm{mmol}$ ) was suspended in freshly distilled $\mathrm{MeOH}(5 \mathrm{~mL})$, a solution of sodium methoxide ( $2 \mathrm{M}, 0.1 \mathrm{~mL}$ ) was added, and the mixture was stirred overnight at rt. TLC showed that the reaction was complete. The resulting solution was de-ionized with Amberlite IR-120 $\left(\mathrm{H}^{+}\right)$anion-exchange resin, filtered, and concentrated. The hexasaccharide 1 was obtained after chromatography on Sephadex $\mathrm{G}-25\left(\mathrm{H}_{2} \mathrm{O}\right.$ solvent) as an amorphous powder $(9.5 \mathrm{mg}, 96 \%)$ after freeze-drying; $[\alpha]_{\mathrm{D}}^{20}-14.9^{\circ} \quad(c 0.1, \mathrm{MeOH}) ; \mathrm{ESMS}$ for $\mathrm{C}_{36} \mathrm{H}_{62} \mathrm{O}_{31}$ (990.86): $989.6[\mathrm{M}-1]^{+}$.

\section{Acknowledgements}

This work was supported by the Chinese Academy of Sciences (Project KJ952J,510) and by the National Natural Science Founda- 
tion of China (Projects 29672049 and 29802009).

\section{References}

[1] (a) J.K. Sharp, B. Valent, P. Albersheim, J. Biol. Chem., 259 (1984) 11312-11320. (b) J.K. Sharp, M. McNeil, P. Albersheim, J. Biol. Chem., 259 (1984), 11321-11336. (c) J.K. Sharp, P. Albersheim, B. Lindberg, J. Biol. Chem., 259 (1984) $11341-11345$.

[2] J.J. Cheorg, W. Birberg, T. Ogawa, M.G. Hahn, Plant Cell., 3 (1991) 127-182.

[3] Synthesis: (a) P. Ossowski, A. Pilotti, P.J. Garegg, B. Lindberg, Angew. Chem., Int. Ed. Engl., 22 (1983) 793794. (b) P. Ossowski, A. Pilotti, P.J. Garegg, B. Lindberg, J. Biol. Chem., 259 (1984) 11337-11340. (c) P. Fugedi, W. Birberg, P.J. Garegg, A. Pilotti, Carbohydr. Res., 164 (1987) 297-312. (d) P. Fugedi, P.J. Garegg, I. Kvarnstrom, A. Pilotti, J. Carbohydr. Chem., 8 (1989) 47-55. (e) P. Fugedi, P.J. Garegg, I. Kvarnstrom, L. Svansson, J. Carbohydr. Chem., 7 (1988) 389-402. (f) N. Hong, T. Ogawa, Tetrahedron Lett., 31 (1990) 31793182. (g) P.J. Lorentzen, B. Helpap, O. Lockhoff, Angew. Chem., Int. Ed. Engl., 30 (1991) 1681-1682. (h) N. Hong, Y. Nakahara, T. Ogawa, Proc. Jpn. Acad., 698 (1993) 55-67. (i) H. Yamada, T. Harad, T. Takahashi, J. Am. Chem. Soc., 116 (1994) 7919-7920. (j) K.C. Nicolaou, N. Winssinger, J. Pastor, F. Derosse, J. Am. Chem.
Soc., 119 (1997) 449-450. (k) S. Aldington, S.C. Fry, Adv. Bot. Res., 19 (1992) 1-101.

[4] W. Wang, F. Kong, Tetrahedron Lett., 39 (1998) 1937 1940.

[5] J.G. Buchanan, J.C.P. Schwarz, J. Chem. Soc., Chem. Commun., (1962) 4770-4777.

[6] M.E. Evans, Carbohydr. Res., 21 (1972) 473-475.

[7] V.I. Betaneli, M.V. Ovchinnikov, N.K. Kochetkov, Carbohydr. Res., 107 (1982) 285-291.

[8] P.J. Garegg, H. Hultberg, Carbohydr. Res., 110 (1982) 261-266.

[9] J. Gass, M. Strobl, P. Kosma, Carbohydr. Res., 244 (1993) 69-84.

[10] W. Wang, F. Kong, J. Org. Chem., 63 (1998) 5744-5745.

[11] C.P.J. Glaudemans, M.J. Bertolini, Methods Carbohydr. Chem., 8 (1980) 271-275.

[12] (a) K.I. Agarwal, A. Yamazaki, P.L. Cashion, H.G. Khorana, Angew. Chem., Int. Ed. Engl., (1972) 451-459. (b) J. Stanêk, Top. Curr. Chem., 154 (1990) 234-242.

[13] X. Ding, W. Wang, F. Kong, Carbohydr. Res., 303 (1997) 445-448.

[14] E. Chelain, S. Czernecki, J. Carbohydr. Chem., 15 (5) (1996) 571-579.

[15] R.R. Schmidt, Angew. Chem., Int. Ed. Engl., 25 (1986) 212-235.

[16] S. Sato, Y. Ito, T. Nukada, Y. Nakahara, T. Ogawa, Carbohydr. Res., 167 (1987) 197-210.

[17] F.-I. Auzanneau, F. Forooghian, B.M. Pinto, Carbohydr. Res., 291 (1996) 21-41.

[18] V.I. Betaneli, M.V. Ovchinnikov, L.V. Backinowsky, N.K. Kochetkov, Carbohydr. Res., 107 (1982) 285-291. 